\title{
Toward Limb Position Invariant Myoelectric Pattern Recognition Using Local-Global Time-Dependent Spectral Features
}

\author{
Rami N Khushaba, Maen Takruri, Jaime Valls Miro, and Sarath Kodagoda \\ All authors are within the School of Electrical, Mechanical and Mechatronics Systems, \\ Faculty of Engineering and Information Technology, University of Technology, Sydney \\ (UTS). \\ Email:Rami.Khushaba@uts.edu.au,Maen.Takruri@uts.edu.au, \\ Jaime.VallsMiro@uts.edu.au,Sarath.Kodagoda@uts.edu.au
}

\begin{abstract}
Recent studies in Electromyogram (EMG) pattern recognition revealed a semantic gap between research findings and a viable clinical implementation of myoelectric control strategies. One of the important factors contributing to the limited performance of such controllers in practice is the variation in the limb position associated with normal use as it results in different EMG patterns for the same movements when carried out at different positions. However, the end goal of the myoelectric control scheme is to allow the amputees to control their prosthetics in an intuitive and accurate manner regardless of the limb position at which the movements are initiated. In an attempt to reduce the impact of limb position on EMG pattern recognition, a new feature extraction method is proposed in this paper that extracts a set of power spectrum characteristics directly from the time-domain with the end goal of forming a set of invariants to limb position. In specific, the proposed method estimates the spectral moments, spectral sparsity, spectral flux, irregularity factor, and signals power spectrum correlation by using Fourier transform properties to form invariants to amplification, translation and signal scaling and provide an efficient and accurate representation of the underlying EMG activity. Additionally, due to the inherent temporal structure of the EMG signal, the proposed method is applied on the global segments of EMG data as well as the sliced segments using multiple overlapped windows. The performance of the proposed features is tested on EMG data collected from eleven subjects, while implementing eight classes of movements, each at five differ-
\end{abstract}


ent limb positions. Practical results indicate that proposed feature set can achieve significant reduction in the classification error rates, in comparison to other methods, with $\approx 8 \%$ error on average across all subjects and limb positions. A real-time implementation and demonstration is also provided and made available at http://youtu.be/JRqXIv0xrN0

Keywords: Electromyogram (EMG), Spectral Moments, Signal Processing.

\section{Introduction}

Human-computer interfaces play a very important role in the advancement of methods enabling humans to interact with and control a specific machine. One of such interfaces directly senses and decodes the Electromyogram (EMG) signals from human muscles and utilizes it in various applications including its use as a source of control for powered prosthetics and rehabilitation devices $[1,2,3]$, speech recognition $[4,5]$, and very recently in developing muscle-computer interfaces for gamers [6, 7]. The control scheme denoted as myoelectric control, employs a pattern recognition approach to discriminate between the EMG signals that belong to different arm movements [1]. The main assumption is that, at a given surface electrode location, the set of parameters, i.e., the extracted features, describing the EMG signal will be more or less the same for a given pattern of muscle activation. In addition, it will also differ from one pattern or mode of muscle actuation to another at the same electrode location [8]. Based on this assumption, successful off-line classification results on pre-recorded signals were reported in the literature $[9,10]$.

Recent interest toward advancing real-time and clinical application of myoelectric control revealed a semantic gap between research findings and a clinically viable implementation [11]. This is mainly driven by several factors that can significantly affect the performance of an EMG pattern classifier and may result in an unusable controller. As an example, Hargrove et al. [12] showed that electrode displacements during usage adversely affect EMG classification accuracy, but training the system to recognize plausible displacement locations can mitigate the effect. The conventionally defined classification accuracy was also recognized as an idealistic measure that may not reflect true clinical performance. Scheme et al. [13] proposed a selective multiclass one-versus-one classification technique allowing for independent adjustment of individual class-pair boundaries making it flexible and 
intuitive for clinical use. Cipriani et al. [14] showed that variations in the weight of the prosthesis and upper arm movements significantly influence the robustness of a traditional EMG classifier causing a significant drop in performance. It was suggested that a robust classifier should add to myoelectric signals some inertial transducers like multi-axes position, acceleration sensors, or sensors able to monitor the interaction forces between the socket and the end-effector. The effects of limb position on pattern recognition based myoelectric control was examined on normal and amputees subjects as another factor [15, 16]. Fougner et al. [17] proposed two possible solutions to reduce the adverse limb position effect including training in multiple limb positions and measuring the position using accelerometers. Additional factors affecting the myoelectric pattern recognition performance could also include minimizing EMG electrode numbers, determining acceptable electrode locations, optimizing electrode recording configurations, and dealing with any other challenges of EMG recording in a dynamic environment [18]. Jiang et al. [19] indicated that unlike the controlled laboratory conditions in fixed arm/trunk positions that exhibit stationary EMG statistical properties, the EMG signal characteristics can easily change in a dynamic environment due to factors like sweat, fatigue, or from different strategies employed by the user who is adapting to the system. The fact that very few myoelectric control systems proposed in the scientific literature can adapt to such changes is by itself a good reason for the lack of usability of these systems in practice. Additional factors were also reported to include: The majority of current pattern classification methods do not provide simultaneous and proportional control, are not implemented with sensory feedback, and do not integrate other sensor modalities to allow complex actions.

The focus of this paper is also targeted on the effect of upper-limb position on EMG pattern recognition as a complementary study to that reported in [17]. We investigate in this paper a new feature extraction method based on local and global spectral characteristics, as an alternative solution to the use of accelerometers, to form a set of invariants to the change in the EMG signals that belong to the same movements. The main arguments here to justify the need for the new feature extraction method include first that the effectiveness of any pattern recognition system is mainly dependent on the quality of the extracted features, and their ability to provide an accurate representation of the underlying activity. Thus, investigating a new feature set to overcome the effects of limb position is of significant importance and should be considered as an initial solution before employing additional inertial sensors. Secondly, 
regardless of the limb position, and the generated EMG activities at the different positions, the EMG pattern classifier should be able to recognize the hand movements accurately. In simpler words, while implementing the same arm or hand movements at different limb positions one would intuitively think that the underlying relation between the EMG activities generated by the different muscles should be the same, to some extent, as these muscles are collaborating to induce the same movement. However, this is not the case as different muscles combinations could be recruited to perform a specific task at different limb positions to stabilize the limb, as indicated by Fougner et al. [17]. Additionally, variations in the underlying EMG activities as induced by the change in the muscle's shape and length while the user attempts to do the same movement at different positions should be captured and recognized as they belong to the same movements class. Such variations can easily change the amplitude, shape, and frequency-domain characteristics of the EMG signal upon what the classifier was initially trained on causing degradation in EMG recognition performance. According to all of the above, we propose a set of power spectrum features as possible invariants to signal amplification, translation and scaling as a possible solution to this problem.

The structure of this paper is as follows: Section 2 first reports a background on EMG feature extraction and then describes the proposed feature extraction method. Section 3 describes the data collection procedure. Section 4 presents the experimental results and finally, conclusions are provided in Section 5.

\section{The proposed Feature Extraction Method}

In an attempt to enhance the performance of an EMG-driven pattern recognition system, a new feature extraction method is presented based on spectral moments and described below. However before proceeding with the description of our proposed method, we proceed first with a background on feature extraction in EMG classification and then link the effect of changing the limb position with the variations in the EMG signal characteristics.

\subsection{EMG Feature Extraction-Literature Review}

Feature extraction addresses the problem of finding the most compact and informative feature set that can accurately describe the EMG signal in a condensed representation. According to Boostani and Moradi [20], for a feature set to be suitable for EMG-based control, the feature space should have a 
maximum class separability, should be robust in a noisy environment as much as possible and should have an associated low computational complexity. To this end, various temporal and spectral approaches to feature extraction were utilized to derive an EMG-based controller [1, 9, 20, 21, 22, 23, 24, 25].

One of the most important advantages of employing time-domain characteristics is the reduction in the complexity associated with the process of feature extraction. Hudgins et al. [1] were among the first to consider time-domain features in myoelectric control while demonstrating the effectiveness of simple features like mean-absolute value, mean absolute value slope, zero crossings, slope-sign changes, and waveform length. Additional methods were also utilized by many research groups including Integral of Absolute Value, Variance, Willison amplitude, the V-order and log detectors, and Root-Mean Square [9, 22, 26, 27, 28]. Although features extracted by time-domain methods proved successful and suitable for real-time control, an argument still exist in the literature that pattern recognition results using these feature vectors may not provide high success rates [29]. This is justified by that such methods assume that the EMG signal is stationary, while it is nonstationary in its nature [3]. This in turn changed the researchers focus to features extracted based on spectral parameters (spectral moments), Autoregressive models $[23,30,31,32,33]$ and time-frequency representation such as the short-time Fourier transform, the Wigner-Ville distribution, the Choi-Williams distribution, the continuous wavelet transform, the wavelet transform, and the wavelet packet transform [2, 23, 34, 35, 36, 37, 38, 39, 40].

Spectral moments appear to be one of the promising approaches for EMG characterization and has been used in several recent attempts [23, 27, 32, 33]. Generally, the first few moments are usually utilized to describe the energy, center frequency, and variance of the EMG power spectrum [23, 21]. Farry et al. [41] indicated that to use EMG spectral features in a real-time control system we need a spectral estimation method that 1) works well on small windows length of EMG data, 2) does not suffer from bias and variance that obscures the spectral features, and 3) incorporates no incorrect assumptions about the structure of the signal. However, it is generally known that if the observed time sequence is too short, the simple spectrogram becomes ineffective due to increased bias and variance while models based on the AR spectrum were reported in some instances to match certain motion artifacts and their output to be badly biased [41, 42]. Du [43] utilized the short-timeThompson-transform to extract the spectral moments and reported advantages upon the short-time-Fourier-transform in terms of classification accu- 
racies of six classes of grasps. Du also reported that the first and the second moments reduced the classification hit rates in certain cases and justified that by the effect of noise on higher order moments but not the lower order ones.

Despite the effectiveness of the aforementioned methods, the long recording lengths (windows) required for spectral analysis and the high computational complexity for time-frequency representations played a significant role for seeking alternative representation of ensemble of simple and complementary set of features with reduced computational requirements upon that of time-frequency methods. As an example, many recent attempts referred to the combination of time-domain features and the autoregressive features, denoted together as TDAR in this paper, as being very efficient and suitable for real-time control $[44,45,46]$. The same combination was also successfully tested in experiments identifying seven user locomotion modes based on myoelectric signals and applied on subjects with long transfemoral (TF) amputations; with results holding promise for future design of neural-controlled artificial legs [47].

Other research groups utilized a combination of spectral moments (zeros, first and second moments) and time domain features [27, 33] reporting enhancements in classification accuracies upon that achieved by individual features only. On the other hand, Vuskovic and Du [32] employed the autocorrelation sequence of the original temporal signal, rather than the signals power spectral density, to estimate a set of efficient and robust to noise moments for EMG classification, i.e., an attempt to provide time-dependent spectral features. In a similar manner, the time-dependent spectral moments represented by Hjorth parameters [48] were recently utilized in EMG classification and showed powerful performance when combined with other features [49]. A significant advantage of these time-dependent spectral moments features is the associated very low computational cost and the good classification performance. However, further investigation on the suitability of the time-dependent spectral moments to problems with different limb positions is missing from the literature and is the main aim of this paper.

In the next section, a new feature set is proposed for EMG classification that can be considered as an extension to Hjorth's approach to describe the frequency-domain contents of a signal from the time-domain perspective. The differences between the proposed features and Hjorth parameters are described as follows: 
- Unlike Hjorth parameters that represent the activity, mobility, and complexity of an EMG trace, the proposed features also measure the sparseness, spectral flux, the irregularity factor (combining information from number of zero crossings, number of peaks and waveform length), and the correlation between the power spectrum of each two signals.

- Unlike Hjorth parameters that consider normalization by higher order moments, the proposed features are normalized by a properly scaled version of the zero order moment. The justification is that higher order moments are not as robust to noise as the low order moments [50].

- Unlike Hjorth parameters, the proposed features are also logarithmically scaled as the power of most physiological features tend to change linearly in the logarithmic scale rather than in the normal scale [51, 52].

\subsection{Factors Affecting EMG Characteristics}

Surface EMG signal characteristics can easily be affected by physical and physiological changes that can make an EMG-driven system unreliable for long-term use. Several factors were identified in the literature to cause variations in the EMG signals including (but not limited too): displacement of the recording electrodes, variation in muscle contraction effort, muscle fatigue, and the corresponding limb position $[19,53]$. All of these factors may affect the stability of time-domain features used for electromyographic pattern recognition. We expand below on the aforementioned factors to better understand how the proposed features in this paper could form invariants for EMG classification.

Under normal conditions, an action potential propagating down a motoneuron activates all the branches of the motoneuron; these in turn activate all the muscle fibers of a motor unit, where a muscle is usually made of several motor units. The membrane depolarization, accompanied by a movement of ions, generates an electromagnetic field in the vicinity of the muscle fibers [54]. An electrode located in this field will detect the potential or voltage (with respect to ground), whose time excursion is known as an action potential. The waveform of an observed action potential will depend on the orientation of the detection electrode contacts with respect to the active fibers. Thus variations in the electrodes recording positions can significantly affect and change the EMG characteristics as the activity of more/less muscles fibers could be captured when the electrodes shift in position, with the 
possibility of picking up the activity of muscle fibers from nearby muscles (muscle crosstalk). The resultant signal at the detection site will constitute a spatial-temporal superposition of the contributions of the individual action potentials. As the activity of additional muscle fibers is picked up by electrodes shift then the shape and the amplitude of the corresponding EMG signal will also change $[54,55]$. On the other hand, it is well known that the muscle contraction force determines the number and type of recruited muscle fibers and the frequency of firing, thus directly affecting the magnitude and frequency of surface EMG signals [53], while muscle fatigue usually causes a power spectrum shift toward low frequencies $[53,56]$. Thus, the effect of the aforementioned factors on EMG can be seen as changes in the EMG amplitude, shape, and frequency shift (spectrum shift toward small frequencies means larger time scale is showing up in the time domain).

The limb position's effect on EMG characteristics can be seen as a combination of several factors that all depend on joint angles, including [17]:

- variations in muscle recruitment for limb stabilization due to gravitational forces, resulting in changes to signal amplitude and the amount of interaction between muscles to perform a specific movement

- electrode shift due to changes in muscle shape, length and position resulting in changes to signal amplitude and frequency as different numbers of muscles fibers could be recruited.

- the force-length relationship of the muscle and the effect of fatigue as a result of variable forces used during sustained contractions, resulting in changes to the signal amplitude and a shift in the frequency spectrum.

Another possible factor to mention here is the effect of employing signal segmentation techniques, overlapping or disjoint windows, for EMG feature extraction. In such a case, the extracted features should be robust against signal translation effect resulting from using small windows sizes required to maintain the system delay within acceptable performance limits, with a maximum of $300 \mathrm{msec}$ defined in the literature [9].

In response to the effect of the above mentioned factors on the EMG characteristics, we present in this paper a new set of power spectrum moments features as a candidate subset to overcome many of the above limitations, that we derive directly from time-domain to preserve computational cost. These features are introduced as invariants to signal translation and timescaling while also attempting to produce a set of features that captures the 
distribution of EMG energy across the different time segments as a proportion of the total record energy regardless of signal amplitude.

\subsection{Feature Extraction Using Time-Dependent Power Spectrum Descriptors}

Assuming a sampled version of the EMG signal record, denoted as $x[j]$, of length $N$ and sampled at $f_{s} \mathrm{~Hz}$, the EMG trace within a certain epoch can be expressed as a function of frequency $X[k]$ by means of Discrete Fourier transform (DFT). Each EMG record is preprocessed by removing the mean and dividing by the range of the data. We start the transformation of the parameters between the frequency and the time domains by observing Parseval's theorem which states that the sum of the square of the function is equal to the sum of the square of its transform

$$
\sum_{j=0}^{N-1}|x[j]|^{2}=\frac{1}{N} \sum_{k=0}^{N-1}\left|X[k] X^{*}[k]\right|=\sum_{k=0}^{N-1} P[k]
$$

where $P[k]$ is the phase-excluded power spectrum, i.e., the result of a multiplication of $X[k]$ by its conjugate $X^{*}[k]$, and $k$ is the frequency index. It is generally well-known that the complete frequency description as derived by means of the Fourier transform is always symmetrical with respect to zero frequency, i.e., it has identical branches stretching into both positive and negative frequencies [48]. As a consequence of this symmetry and because we have no direct access to the power spectral density from the time-domain then we are left with the option of dealing with the whole spectrum, including positive and negative frequencies. Thus, in a statistical approach to the shape of the frequency distribution, all odd moments will become zero, according to the definition of a moment $m$ of order $n$ of the power spectral density $P[k]$ which is given by

$$
m_{n}=\sum_{k=0}^{N-1} k^{n} P[k]
$$

In the above equation, when $n=0$ we will make use of Parseval's theorem in Eq.1, and for most of the rest of the value of $n$ we will use the timedifferentiation property of the Fourier transform. Such a property simply states that the $n$ 'th derivative of a function in the time-domain, denoted as $\triangle^{n}$ for discrete time signals, is equivalent to multiplying the spectrum by $k$ raised to the $n$ 'th power 


$$
\mathcal{F}\left[\triangle^{n} x[j]\right]=k^{n} X[k]
$$

To this end, we define the features utilized in this paper as the logarithmically scaled versions of the following properties as shown in Fig.1:

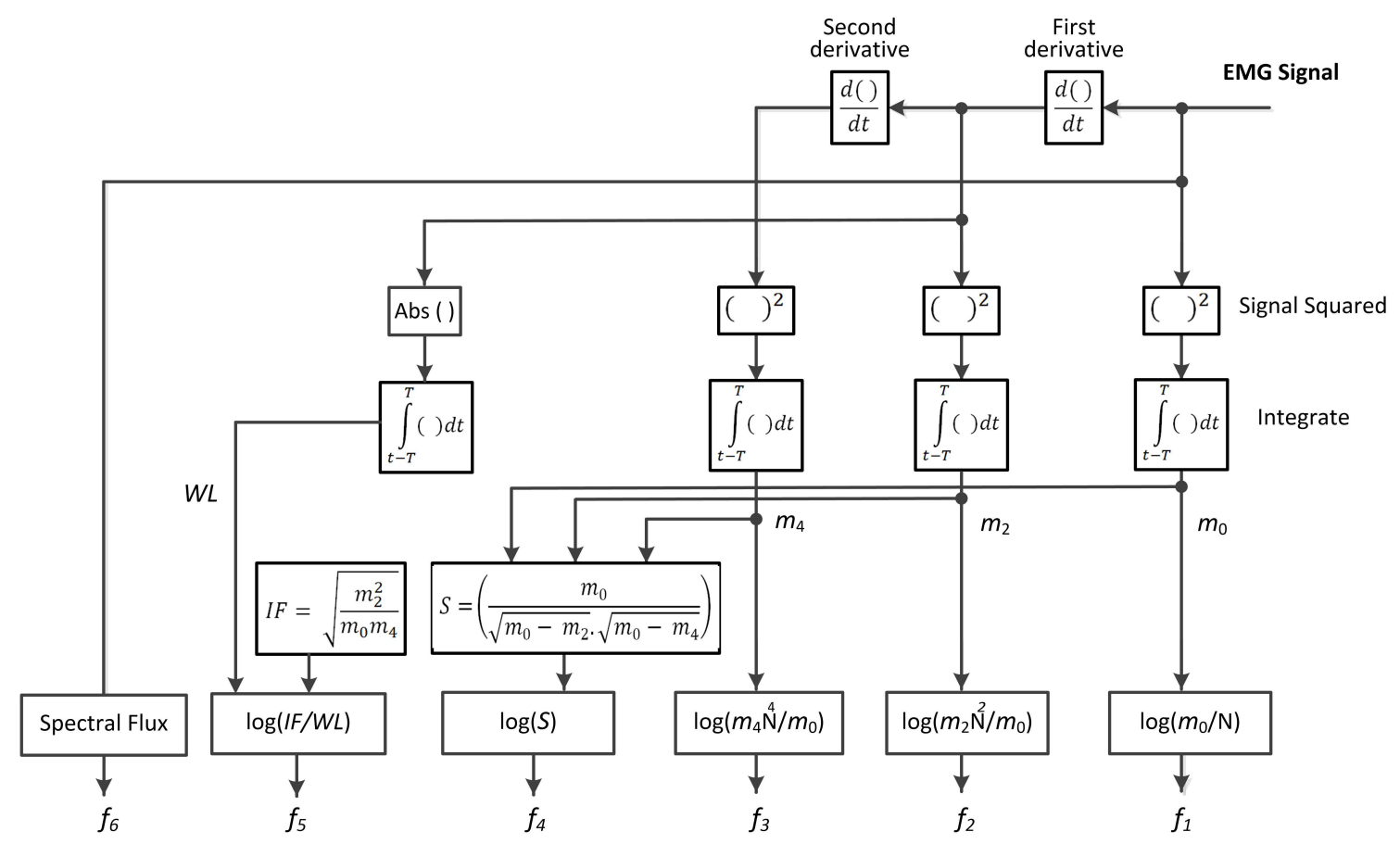

Figure 1: Schematic diagram of the proposed feature set.

- zero order moment $\left(m_{0}\right)$ : which is an indicator of the total power in the frequency domain. We divided this feature by the signal length to form an indicator of the signal power that is invariant against the change in the signal scale (time scale and not amplitude scale).

$$
\log \left(\frac{m_{0}}{N}\right)=\log \left(\frac{\sum_{k=0}^{N-1} k^{0} P[k]}{N}\right)=\log \left(\frac{\sum_{j=0}^{N-1}|x[j]|^{2}}{N}\right)
$$

- normalized second and fourth order moments: according to Hjorth [48] the second moment can be considered as a power, but 
then of another spectrum $k^{2} P[k]$, corresponding to a frequency function $k . X[k]$ and given as

$$
m_{2}=\sum_{k=0}^{N-1} k^{2} P[k]=\sum_{k=0}^{N-1}(k X[k])^{2}=\sum_{j=0}^{N-1}(\triangle x[j])^{2}
$$

A repetition of this procedure gives the moment.

$$
m_{4}=\sum_{k=0}^{N-1} k^{4} P[k]=\sum_{j=0}^{N-1}\left(\triangle^{2} x[j]\right)^{2}
$$

According to Flusser et al. [50], scale-invariance property is achieved by proper normalization of low-order moments as they are more stable to noise and easier to calculate than higher order moments. Thus, our second feature is defined as $\log \left(\left|m_{2} N^{2} / m_{0}\right|\right)$ and third feature as $\log \left(\left|m_{4} N^{4} / m_{0}\right|\right)$.

- Sparseness: this measures quantifies how much energy of a vector is packed into only a few components and is given in this paper as

$$
S=\log \left(\left|\frac{m_{0}}{\sqrt{m_{0}-m_{2}} \cdot \sqrt{m_{0}-m_{4}}}\right|\right)
$$

such a feature describes a vector with all elements equal with a sparseness measure of zero, i.e., $m_{2}$ and $m_{4}=0$ due to differentiation and the $\log \left(m_{0} / m_{0}\right)=0$, whereas for all other sparseness levels it should have a value bigger than zero. The $m_{4}$ moment was added to this feature as an indication of the rate of change of $m_{2}$ itself.

- Irregularity Factor (IF): which is defined as the number of upward zero crossings divided by the number of peaks. The number of upward zero crossings (ZC) and the number of peaks (NP) in a random signal can be expressed solely in terms of their spectral moments as given below [57]

$$
Z C=\sqrt{\frac{m_{2}}{m_{0}}}
$$




$$
N P=\sqrt{\frac{m_{4}}{m_{2}}}
$$

The irregularity factor is defined as the ratio of zero crossing over number of peaks and is given as [57]:

$$
I F=\sqrt{\frac{m_{2}^{2}}{m_{0} \cdot m_{4}}}
$$

We propose in this paper a modified irregularity factor that divides the above measure in Eq.10 by the waveform length to account for the ratio of ZC over NP within a specific waveform length rather than dividing by the record length as in [57].

- Spectral Flux: which measures the local spectral change between consecutive samples in the power spectral density [61]

$$
S F=\sum_{k=0}^{N-1}\left(\sqrt{P[k]}-\sqrt{P\left[k_{o}\right]}\right)^{2}
$$

where $P\left[k_{o}\right]$ is a frequency shifted version of $P[k]$ and can also be written as $P\left[k-k_{o}\right]$. Referring to Eq.1 it is clear that $P[k]=|X[k]|^{2} / N$ and that $P\left[k_{0}\right]=\left|X\left[k-k_{0}\right]\right|^{2} / N$. Hence, the spectral flux equation can be written as follows:

$$
\begin{array}{r}
S F=\frac{1}{N} \sum_{k=0}^{N-1}|X[k]|^{2}-\frac{2}{N} \sum_{k=0}^{N-1}\left(X[k] X\left[k-k_{o}\right]\right) \\
+\frac{1}{N} \sum_{k=0}^{N-1}\left|X\left[k-k_{0}\right]\right|^{2}
\end{array}
$$

Using Parserval's theorem and the shifting theorem, we can re-write (12) as follows: 


$$
\begin{array}{r}
S F=\sum_{n=0}^{N-1}|x[n]|^{2}-\frac{2}{N} \sum_{k=0}^{N-1}\left(X[k] X\left[k-k_{o}\right]\right)+ \\
\sum_{n=0}^{N-1}\left|x[n] e^{j 2 \pi k_{o} n / N}\right|^{2}
\end{array}
$$

Denoting $X\left[k-k_{o}\right]$ by $Y[k]$ which implies that $y[n]=x[n] e^{j 2 \pi k_{o} n / N}$ :

$$
S F=\sum_{n=0}^{N-1}|x[n]|^{2}-\frac{2}{N} \sum_{k=0}^{N-1}(X[k] Y[k])+\sum_{n=0}^{N-1}|y[n]|^{2}
$$

Now working on the middle term we get:

$$
\begin{aligned}
X[k] Y[k] & =\sum_{m=0}^{N-1}(x[m]) e^{-j 2 \pi m k / N} Y[k] \\
& =\sum_{m=0}^{N-1}(x[m]) \sum_{n=0}^{N-1}(y[n-m]) e^{-j 2 \pi n k / N} \\
& =\sum_{n=0}^{N-1} \sum_{m=0}^{N-1}(x[m])(y[n-m]) e^{-j 2 \pi n k / N} \\
& =\sum_{n=0}^{N-1}(x * y)_{n} e^{-j 2 \pi n k / N} \\
& =D F T_{k}(x * y)
\end{aligned}
$$

substituting back into (14):

$$
\begin{array}{r}
S F=\sum_{n=0}^{N-1}|x[n]|^{2}+\sum_{n=0}^{N-1}|y[n]|^{2} \\
-\frac{2}{N} \sum_{k=0}^{N-1} \sum_{n=0}^{N-1} \sum_{m=0}^{N-1}(x[m])(y[n-m]) e^{-j 2 \pi n k / N}
\end{array}
$$


and replacing $y[n]$ by $x[n] e^{j 2 \pi k_{o} n / N}$ :

$$
\begin{aligned}
\text { rcl } & S F=\sum_{n=0}^{N-1}|x[n]|^{2}+\sum_{n=0}^{N-1}\left|x[n] e^{j 2 \pi k_{o} n / N}\right|^{2} \\
& -\frac{2}{N} \sum_{k=0}^{N-1} \sum_{n=0}^{N-1} \sum_{m=0}^{N-1}(x[m]) \cdots \\
& \left(x[n-m] e^{j 2 \pi k_{o}(n-m) / N}\right) e^{-j 2 \pi n k / N}
\end{aligned}
$$

which in turn can be written as

$$
\begin{aligned}
& S F=2 \sum_{n=0}^{N-1}(x[n])^{2}-\frac{2}{N} \times \\
& \sum_{k=0}^{N-1} \sum_{n=0}^{N-1} \sum_{m=0}^{N-1}(x[m])(x[n-m]) e^{-j 2 \pi n k / N}
\end{aligned}
$$

which is equivalent to:

$$
S F=2 \sum_{n=0}^{N-1} x[n]^{2}-\frac{2}{N} \sum_{k=0}^{N-1} \sum_{n=0}^{N-1}(x * x)_{n}
$$

When implementing this feature in the frequency-domain, different spectral flux functions were proposed in the literature using $L_{1}$-norm or $L_{2}$-norm with attempts to smooth this feature by the rectification of the difference between the samples, employing hamming windows before utilizing FFT, or employing smoothing kernels (a discussion is provided by Bello et al. [58]). As we are implementing this feature from the time-domain then rectifying the spectral difference between the samples is not an option. However, we do employ hamming windows in the later sections to acquire the local moments as will be discussed, which further smooth this feature.

An example of applying the proposed features on different versions of the wavelet Morlet function is shown in Fig.2. The Morlet function 
was chosen here as a simple example that can be easily reproduced by the reader. One can clearly see that despite the different scales, signal amplitudes and dc-levels in these signals, the extracted features were almost exactly the same. This in turn indicates the capabilities of the proposed feature set as an invariant descriptor to the changes encountered in the signal.
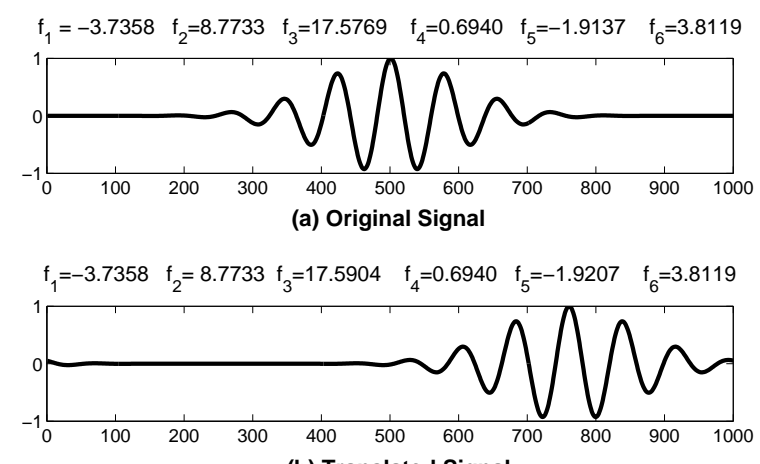

(b) Translated Signal

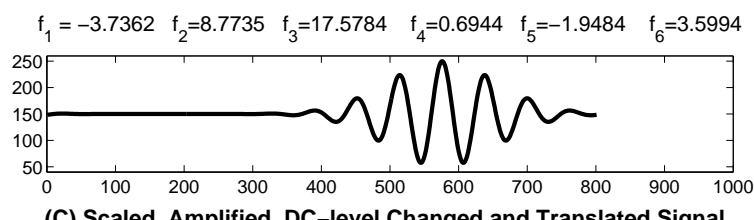

(C) Scaled, Amplified, DC-level Changed and Translated Signal

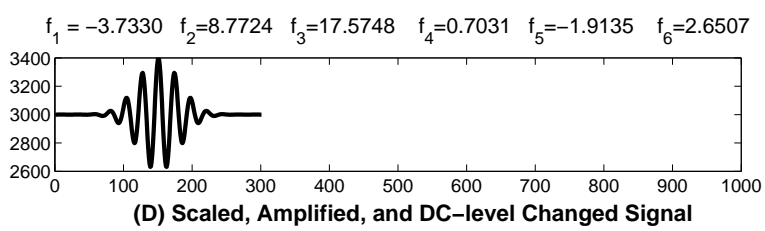

Figure 2: An example of applying the proposed features on different versions of the Morlet function (different scales, signal amplitudes, and dc-levels).

- The final feature proposed here is denoted as the "spectrum correlation"' that is applied on the global windows and could be seen as similar in its context to the well-known measure of spectral coherence [59]. Unlike the coherence measure of similarity that requires access to the power spectrum of the signals, the proposed measure attempts to capture the similarity between the power spectrum of two signals from the time-domain directly by using the cross-correlation 
and Fourier transform relations. In signal processing, cross-correlation is a measure of similarity of two waveforms as a function of a time-lag applied to one of them. Cross-correlation is a very similar operation to convolution, except that the "kernel" is not time-reversed during the operation. In the time-domain point of view, the cross-correlation theorem states that the Fourier transform of the cross-correlation of two signals is equal to the product of the individual Fourier transforms [60], where one of them has been complex conjugated:

$$
x \star g \Leftrightarrow \bar{X} . G
$$

where $X[k]$ and $G[k]$ are the frequency domain representations of the signal $x[n]$ and $g[n]$ by using the DFT. However, since we are interested in having an estimate of the similarity of the frequency domain representations of the two signals then we simply reverse the relation in Eq.20 and multiply the time domain signals to get a cross-correlation in the frequency domain. In simpler words, our proposed feature would be

$$
C_{x, g}=\frac{\sum_{n=0}^{N-1} x[n] \cdot \bar{g}[n]}{\sqrt{\sum_{n=0}^{N-1} x[n]^{2}} \times \sqrt{\sum_{n=0}^{N-1} g[n]^{2}}}
$$

The complex conjugate in the frequency domain maps to a time reversal in the time-domain which is represented by the reversed version of $g[n]$ that we denoted as $\bar{g}[n]$. On the other hand, the measure was normalized by the energy of the individual signals to limit the range of the feature between 0 and 1 while describing the degree of synchrony between the two signals.

\subsection{Local-Global Time-Dependent Spectral Moments}

According to Du [43], the energy of an EMG signal is usually not evenly distributed and that the variation of energy upon time contains the most important attributes for a muscle's movement. In this paper, the raw EMG signal is divided into 3 segments and each segment is multiplied with a windowing function, selected as a hamming window for simplicity (Trapezoidal windows provided similar results) as shown in Fig.3. The proposed feature extraction method is then applied on each of these local segments of EMG as 
well as on the total EMG data within the epoch. It should be mentioned here that the zeros order moments of the local segments were divided by the zero order moment or their corresponding total record to provide relative energy features that are invariants to amplitude change.

Given the six features extracted from each segment and the existence of four segments (3 local and one global), then this in turn results in 24 features per each EMG epoch from each EMG channel ( 24 features $=6$ features per segment $\times 4$ segments). The total number of features from 7 channels was then calculated as 24 features/channel $\times 7$ channels $=168$ features in total.

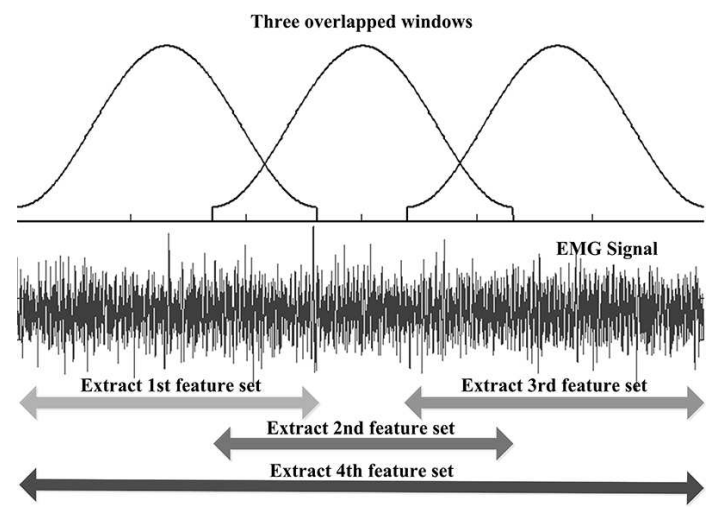

Figure 3: A schematic of the local-global moments extraction from each EMG segment.

In order to account for the relation between the different EMG channels as well as the individual EMG channels activities estimated above, then we have applied our proposed spectrum correlation measure on all possible permutations of existing channels resulting in the correlation values between each possible two channels. Thus, the final number of features is 168 features (24 feature per channel, from 7 channels) +21 features (spectrum correlation between each two channels, in a 7 channels problem) $=189$ features.

\section{Data Collection}

Eleven subjects, nine males and two females, aged between 20-37 years were recruited to perform eight classes of motion. All participants provided informed consent prior to participating in the study as was approved by the university research ethics committee. Despite the fact that the recruited subjects were all normally limbed and had no neurological or muscular disorders, one can easily notice that the same approach can apply for transradial 
amputees. The main argument here is that we are proposing an EMG based control scheme that is not only intended for amputees and prosthetics use but can also be applied on various EMG based control applications (including Microsoft's muscle-computer interfaces for gaming). However, for transradial amputees, the muscles in the residual forearm physiologically used for flexing/extending the hand fingers and wrist are the most appropriate targets for multifingered prostheses control. Thus, the datasets were recorded using seven EMG channels (Delsys DE 2.x series EMG sensors) mounted across the circumference of the forearm and processed by the Bagnoli desktop EMG system from Delsys Inc., as shown in Fig.4. This in turn justifies the clinical relevance of the proposed scheme as it also applies on transradial amputees. The first EMG sensor was placed on the Palmaris Longus muscle on the right hand of each participant while the rest of the sensors were placed in a way that guarantees an equal spacing between the adjacent sensors. A 2-slot adhesive skin interface was applied on each of the sensors to firmly stick them to the skin. A conductive adhesive reference electrode, dermatrode reference electrode, was placed on the shoulder of each of the subjects during the experiments. The collected EMG signals were amplified using a Delsys Bagnoli-8 amplifier to a total gain of 1000. A 12-bit analog-to-digital converter (National Instruments, BNC-2090) was used to sample the signal at $4000 \mathrm{~Hz}$; the signal data were then acquired using Matlab software from Mathworks ${ }^{1}$. The EMG signals were then bandpass filtered between 20-450 $\mathrm{Hz}$ with a notch filter implemented to remove the $50 \mathrm{~Hz}$ line interference.

Five different limb positions were considered in this research as shown in Fig.5. The selected limb positions were chosen to cover most of the positions at which a subject may implement his/her daily activities, including situations where the test subject's arm was hanging straight down hanging at the side (as shown in position P1), the arm was straight reaching forward (as shown in position $\mathrm{P} 4$ ), the arm was reaching up in an angle of 45 degrees (as shown in position P5), and two more possible situations between P1 and P4 (as shown in positions P2 and P3). A set of eight hand motions was performed by each subject at each of these limb positions, including: wrist flexion (1), wrist extension (2), pronation (3), supination (4), power grip (5), pinch grip (6), open hand (7), and rest (8) as shown in Fig.6. These are the same limb positions and hand movements implemented in $[15,16,17]$ and

\footnotetext{
${ }^{1}$ www.mathworks.com
} 


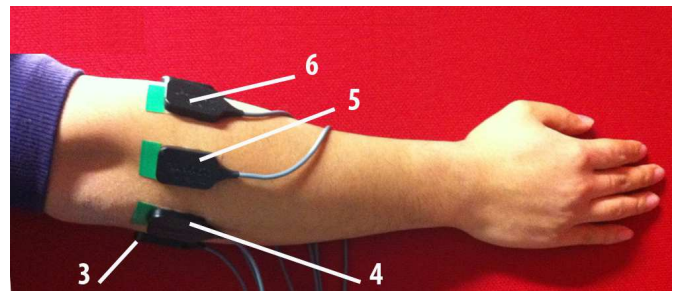

(a) Posterior electrodes positions

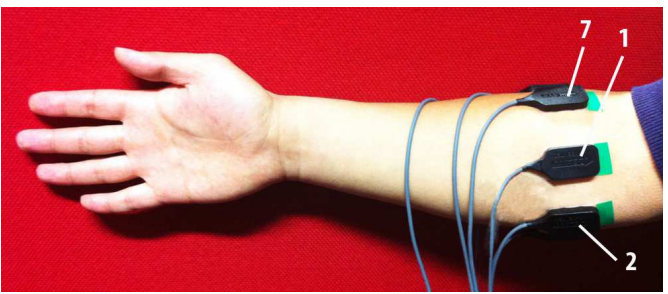

(b) Anterior electrodes positions

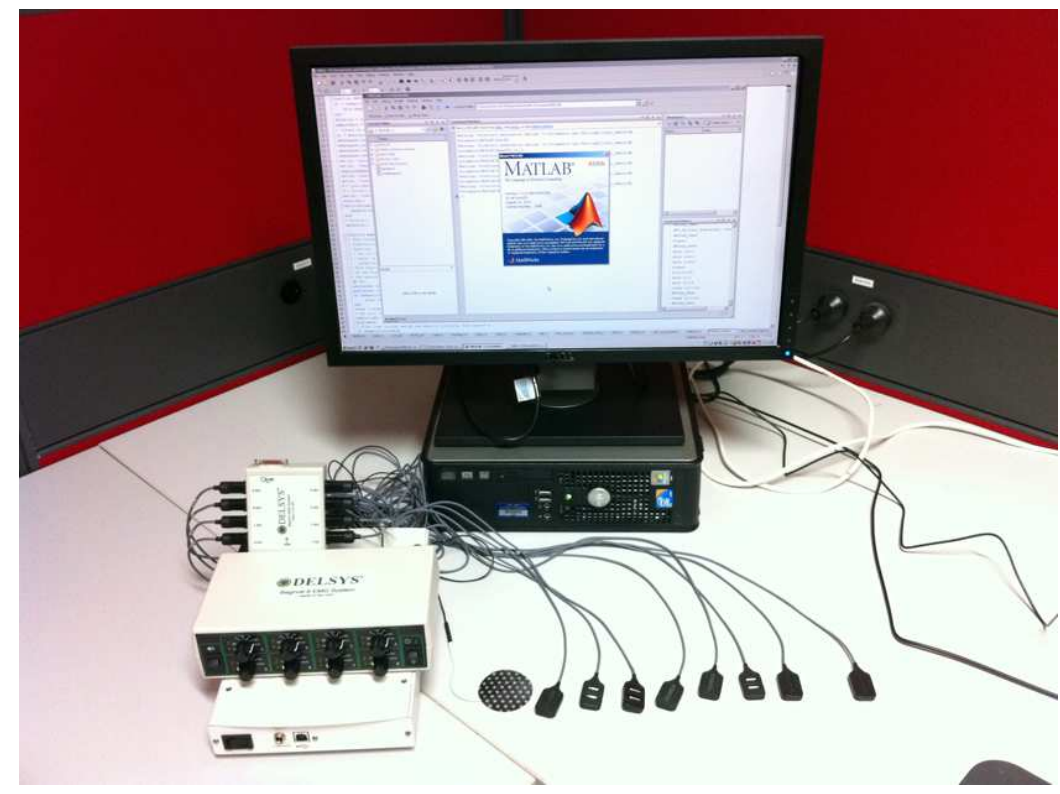

(c) The EMG System hardware utilized in experiments.

Figure 4: EMG system hardware utilized in this research.

were chosen to provide competent experiments and complimentary results, but without using accelerometers. Auditory and visual cues instructed the participants on the required movement and the starting and ending points at which each of the hand movements was implemented in each trial. In specific, the data collection program showed each participant an image of the requested hand movement and the number of trials collected so far while also informing the subjects with audio cues about when to start and when to stop the specific hand movement. As the data is collected in a discrete manner, no overlapping among EMG of different classes can happen in the training phase, while in the testing phase the classifier assigns each of the testing samples 
to one of the predefined hand movements. Six trials of each motion at each limb position were acquired while each motion was sustained for a period of 5 sec only within each trial, with a resting period of 3-to- 5 sec given between trials. These trials were equally divided into 3 trials allocated for training the classifiers and 3 trials allocated for testing. Depending on the number of trials per each motion and the length of each recording trial, then the amount of extracted data from each position could be significantly large to allow the classifier to generalize well on the testing data. In specific, given $5 \mathrm{sec}$ of collected data per each trial sampled at $4000 \mathrm{~Hz}$ and a window size of $100 \mathrm{msec}$ incremented by $25 \mathrm{msec}$, then the number of samples extracted from each trial can be calculated as $(5 * 4000-100 / 1000 * 4000) /(25 / 1000 * 4000)+1=197$ samples from each trial of each hand movement. In a problem with 3 trials allocated for training and another 3 trials allocated for testing, then the number of samples per each movement in each position would be $197^{*} 3=591$ samples per each movement for training and another 591 samples per each movement for testing. For a problem with 8 classes of hand movements the total number of training samples would be $591 * 8=4728$ samples for training and 4728 samples for testing. This in turn explains the distribution of the training and testing data from each position.

When testing the performance of the system, experiments were performed to included training on single limb position and training on multiple limb positions. In the latter case, the features extracted from the EMG data of four limb positions were utilized for training while the features extracted from the fifth position (unseen during training) were used for testing. In this manner, the data from one completely unseen position is utilized for testing to validate the generalization capability of the proposed system while implementing the same movements at a different limb position.

On the other hand, our experiments also included a real time testing

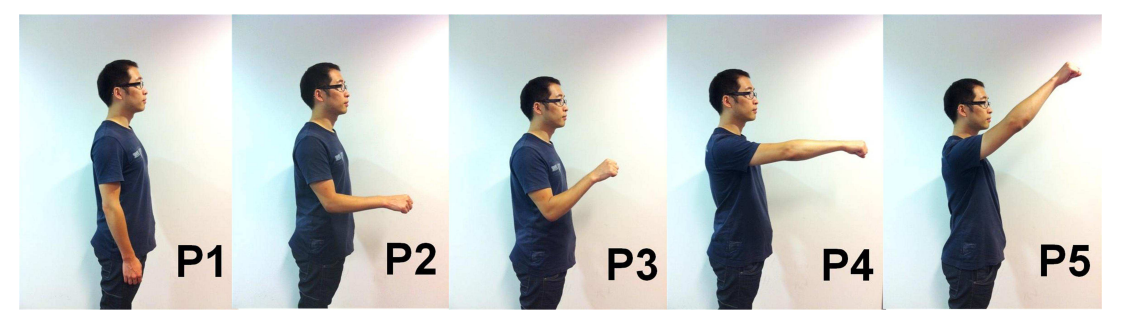

Figure 5: Various limb positions considered in this research. 
session in which the classifier was trained on data from three limb positions only and tested on different limb positions, including those completely unseen during training, as will be described in the later sections.

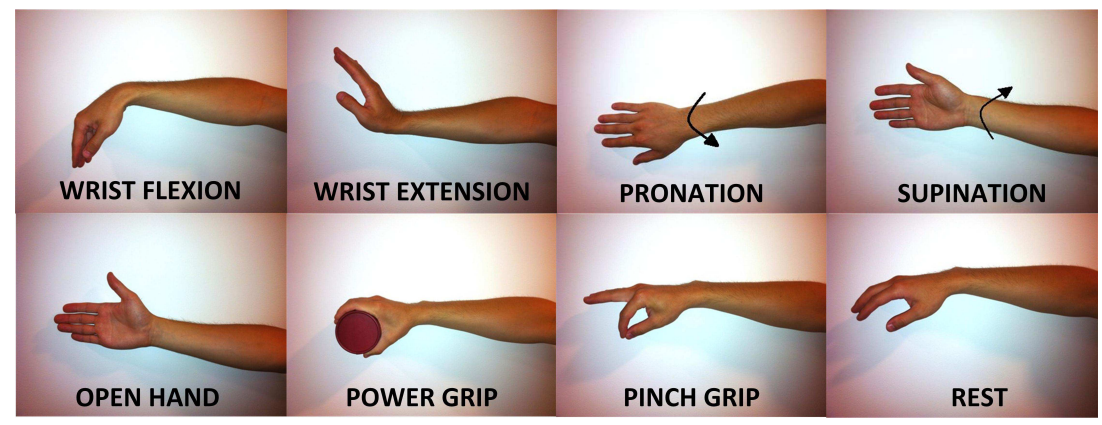

Figure 6: Different classes of hand movements considered in this research, these were repeated at each of the aforementioned limb positions.

\section{Experiments and Results}

Before proceeding with the description of the experimental results, we list first the details of the pattern recognition system employed within the experiments as given below

- Feature Extraction and Reduction: In the feature extraction process, the EMG data was sliced into global segments of 100 msec spaced by $25 \mathrm{msec}$ from the next segment, i.e., a sliding window approach with 100 msec window size advanced by $25 \mathrm{msec}$ each time to get the next window. The rationale for the choice of the $100 \mathrm{msec}$ window size is that it is generally well-known that the total delay in any EMG-driven system needs to be kept below 300 msec to achieve real-time control $[36,9]$. However, there is a current debate on this figure in the literature with some research groups indicating that $300 \mathrm{msec}$ is too long and that this should range within 100-125 ms [62]. Thus, we opt to keep the window length of 100 msec to fit within the aforementioned figures. Each window of $100 \mathrm{msec}$ was further divided using the aforementioned 3 windows technique to produce 24 features per window as mentioned previously. In order to test the effectiveness of the proposed feature set, we divided the experiments into distinct sections in a similar manner 
to that performed by Fougner et al. [17]. The dimensionality of the extracted feature set with 189 features was reduced using the orthogonal fuzzy neighborhood discriminant analysis (OFNDA) feature projection method that was recently proposed by Khushaba et al. [45]. OFNDA is a variation of the classical Fisher discriminant analysis (LDA) feature projection technique and it similarly maps the original feature set into a new domain with $k$-1 features only, with $k$ being the number of classes, i.e., 7 features in our problem.

- Classification methods: A set of four different classifiers were utilized in the experiments including: a support vector machine classifier (LIBSVM library ${ }^{2}$ ) was utilized due to its effectiveness in EMG classification [9, 63], Linear Discriminant Analysis (LDA) [9], $k$-Nearest Neighbor classifier $(k \mathrm{NN})$ with $\mathrm{k}=5$, and Extreme Learning Machine (ELM) library ${ }^{3}$ with the number of hidden nodes in the hidden layer equal to 780 (chosen empirically) [64]. The complexity parameter C of the SVM classifier, as well as the gamma parameter in the kernel function, were optimized for each subject and each position within the range $-4 \leq \log _{10}(\mathrm{C}) \leq 4$. The default implementation of the LIBSVM classifier includes a one-versus-one implementation while we have also implemented the one-versus-rest approach. However, an analysis of variance (ANOVA) test with a significance level of 0.05 indicated that there were no statistical significant differences between the results from both of these approaches, with $p$-values $>0.05$. Thus, we opted to use the default implementation of the LIBSVM classifier with one-versusone approach.

- PostProcessing: On the other hand, the outputs of the classifiers were smoothed using a majority voting post-processing step while looking at the decisions of the previous 8 windows to smooth the current one. Majority voting was selected upon other postprocessing steps, like the Bayesian fusion presented by Khushaba et al. [65], due to two reasons. These include: firstly that we are using an overlapping windowing scheme in this paper while the Bayesian fusion requires the use of a disjoint windowing scheme, and secondly that our work is presented as

\footnotetext{
${ }^{2}$ www.csie.ntu.edu.tw/ cjlin/libsvm/

${ }^{3}$ www.ntu.edu.sg/home/egbhuang/elm_codes.html
} 
a complementary study to that reported in [17] with majority voting. Thus we opt to use majority voting to provide a fair comparison.

A scatter plot of the EMG features extracted from the different limb positions for one subject were first observed to visually inspect the distribution of the extracted features across the different limb positions, these are shown in Fig.7. These plots were made upon the most discriminant three components after the dimensionality reduction method with OFNDA [45], as otherwise one would not be able to plot the scatter of the full feature set. Despite the almost consistent distribution of the features across the different classes in the training set at each position, however, there are some changes to the distribution of the features across positions and the within class variance in each position, especially at position 3. This could be justified by the variations in muscle recruitment for limb stabilization at each position. Thus, unless training data is acquired from several positions then the classifier would not be able to generalize well on unseen new positions.

In the next section, the hypothesis that training data should be acquired from multiple positions will be tested while also observing the best positions from which the training data should be acquired. Thus, we have divided the experiments into three main sections: the first included experiments in which the training data is acquired from a single limb position and testing data from multiple limb positions. The second set of experiments included training the classifiers with data from multiple limb positions and testing on the data acquired from a completely unseen position during training, i.e., training and testing positions are independent. Finally, the third section included in the experiments was devoted to the comparison with other feature sets from the literature.

\subsection{Training in a single limb position}

In the first part of the experiments, we train each of the different classifiers with the EMG features from each of the limb positions individually and then test the capability of the trained classifier on the generalization upon unseen data from all possible positions, i.e., training and testing were performed on all possible permutations of the different positions. As an example, the first row in Fig. 8.a shows the classification error rates when training the LIBSVM classifier with the extracted EMG features from P1 only (training positions reported in the rows) and then testing the LIBSVM classifier with new data from all possible positions (testing positions reported in the 


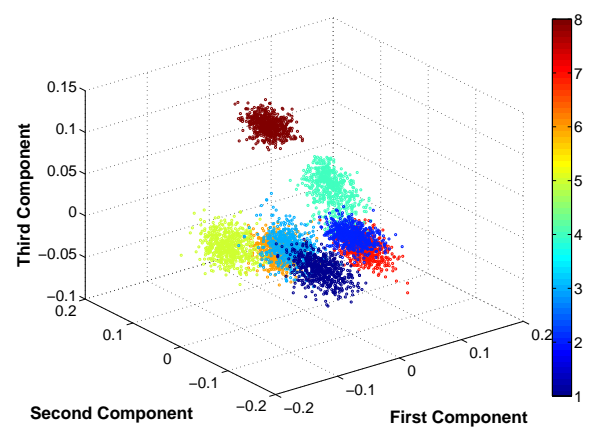

(a) Position 1

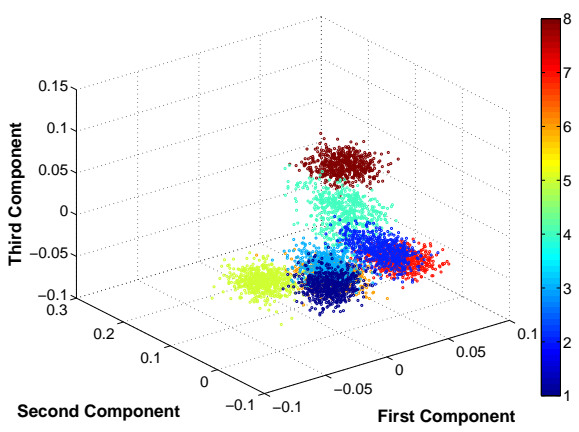

(b) Position 2

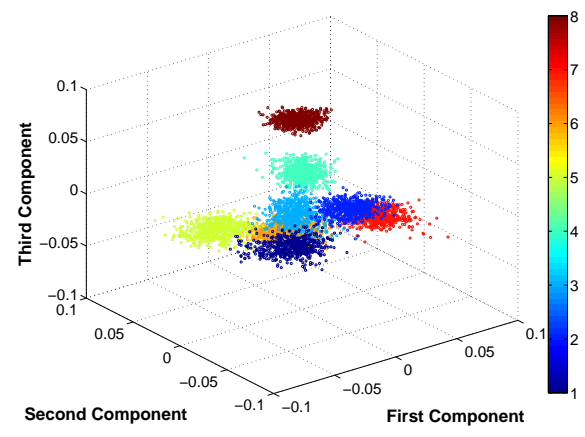

(c) Position 3

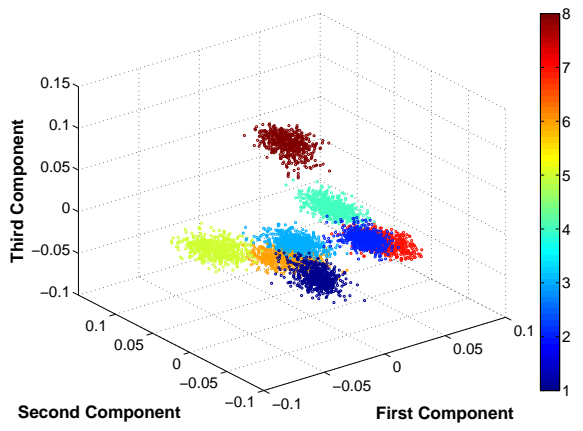

(d) Position 4

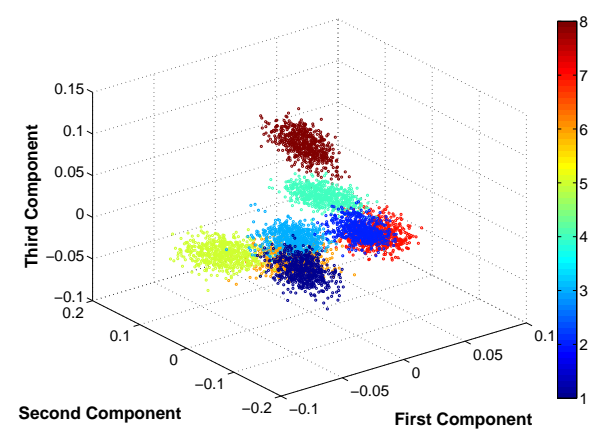

(e) Position 5

Figure 7: Scatter plots of the extracted first three discriminant components with OFNDA across the different limb positions. Colorbar variations in color refer to the problem classes, these are indexed from 1 to 8 . 
columns). These results represent the average across all problem classes while training the classifier with data from P1 and then testing the classifier with data from each of the corresponding limb positions reported in the columns. The results in the second row show the classification error rates when training the LIBSVM classifier with the EMG features from P2 while testing data is acquired from all possible positions, and so on for the rest of the rows. Each entry in these error matrices represents the average error of all motion classes across all subjects for the indicated training and test positions with standard error of mean reported between brackets. The classification errors shown in the main diagonal represent the intra-position classification errors, while the off-diagonal elements represent the inter-position errors. The mean intraposition classification errors (on the diagonal) were $1.68 \%, 2.33 \%, 1.79 \%$, and $1.98 \%$ respectively for the LIBSVM, LDA, $k \mathrm{NN}$, and ELM classifiers whereas the mean inter-position errors were $22.58 \%, 24.11 \%, 22.49 \%$ and $24.68 \%$ respectively for the LIBSVM, LDA, $k \mathrm{NN}$, and ELM classifiers. These results clearly indicate that firstly all of the classifiers achieved nearly similar performance with the proposed features due to the robustness of the proposed feature set against the change of the classifiers. Additionally, both of the LIBSVM and the $k \mathrm{NN}$ classifiers have the potential to perform slightly better than other classifiers in this problem as they tend to produce lower error rates. However, given the computational requirements of the $k \mathrm{NN}$ classifier that compares each testing sample to all of the stored training samples then one might prefer to utilize the LIBSVM classifier.

As expected, one can clearly notice the effect of the different limb positions on the accuracy of the myoelectric pattern recognition system. In such a case, the classifier is only able to generalize well on the data that belongs to the same limb position that the classifier was originally trained on, i.e., EMG classification error is strongly dependent on limb position. As a result, training a prosthetic control system in a single position may be insufficient to develop a system that can perform well in multi-position usage. Thus, more training data from multiple EMG recording positions is obviously required as was indicated by Chen et al. [16]. On the other hand, Fougner et al. [17] and Scheme et al. [15] suggested the additional use of accelerometers to further reduce the classification error rates while training on data from multiple limb positions.

In order to further support the previous suggestion on the need to train the data from multiple limb positions, the confusion matrices were also computed on all possible permutations of the different positions. Since all clas- 


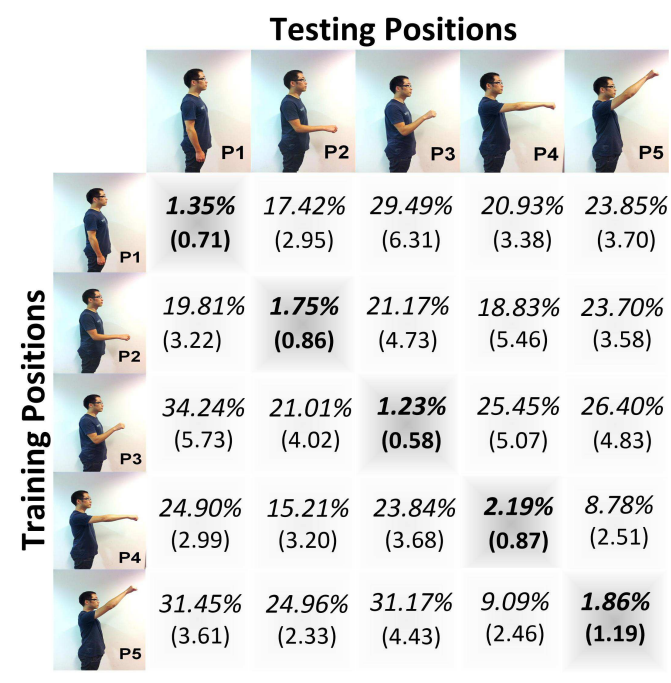

(a) Using LIBSVM classifier

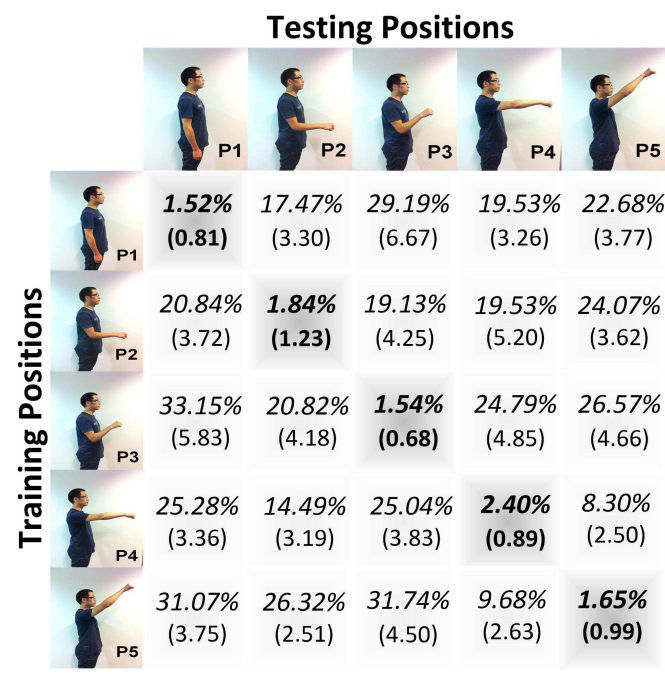

(c) Using $k \mathrm{NN}$ classifier

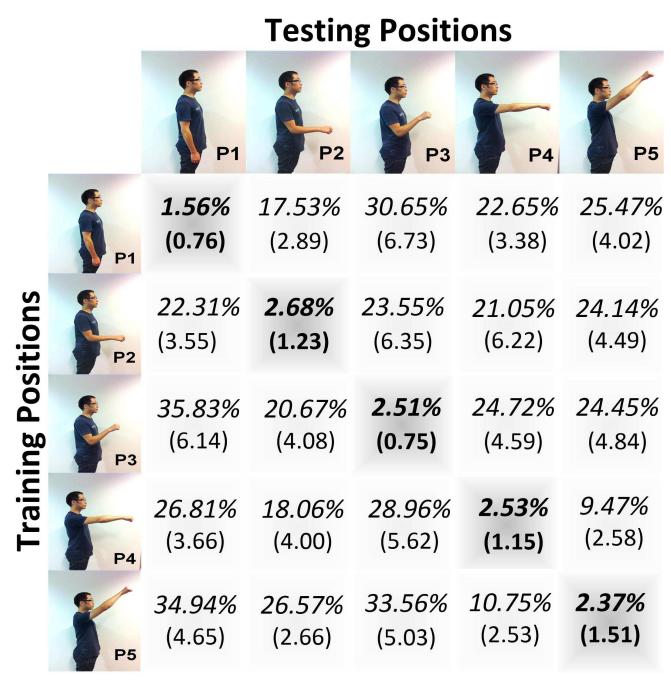

(b) Using LDA classifier

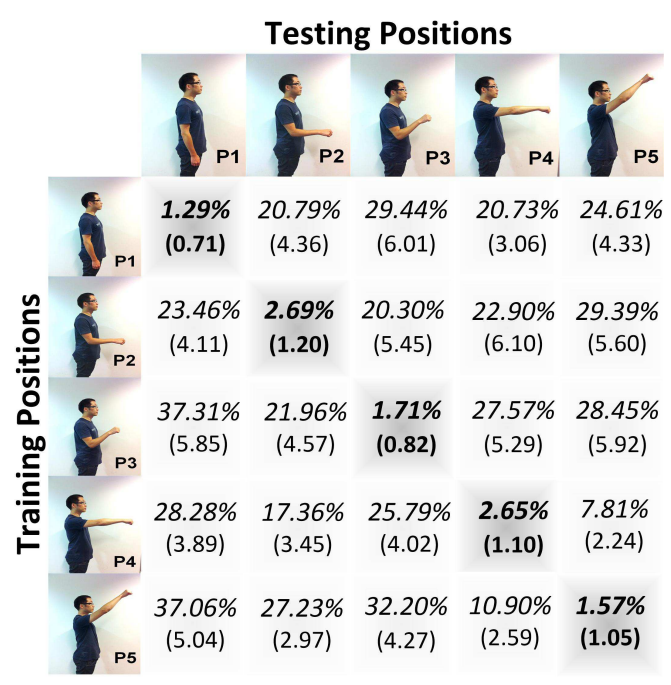

(d) Using ELM classifier

Figure 8: Inter-position classification error (in \%), averaged across all subjects and classes of hand movements and reported as mean error\% (standard error of mean).

sifiers presented nearly similar classification results, we opted to present the confusion matrices plots using only the LIBSVM classifier as shown in Fig.9, representing the average across all subjects, as the LIBSVM classifier per- 
formed slightly better than other classifiers in terms of error rates reduction, though no statistical significant differences were observed in the classification error rates (using ANOVA with significance level of 0.05, the achieved $p$-values were all $>0.05)$. The confusion matrices plots assert the significance of the intra-position classification results and further suggest that there are particular grasps/hand movements that are easier to recognize than others. As an example, wrist extension (class-2) and power grip (class-5) seems easy to recognize when training data is acquired from almost any limb position while testing on any possible position. However, there are also different movements that are harder to recognize across different limb positions, for example training on $\mathrm{P} 1$ and testing on $\mathrm{P} 3$ highly reduces the recognition rates of wrist flexion (class-1) and pinch grip (class-6), while training on P4 and testing on P1 highly affects the recognition rate of supination (class-4), pinch grip (class-6), and open hand (class-7). Thus, there is a clear relation between the limb positions from which the training data is acquired and the recognition rates of the different movements performed at different limb positions.

In the next section, we test the hypothesis that adding training data from multiple limb positions will enhance the classifier performance. This is justified by the fact that the classifier is made aware of the distribution of the data at different limb positions and should therefore be able to better generalize on unseen data.

\subsection{Training in multiple limb positions}

Five different testing situations are proposed each corresponding to one limb position being held for testing, i.e., the data from each of the five positions (P1, P2, P3, P4, and P5) will be utilized as a testing position while training on all other positions. As an example, when the data from $\mathrm{P} 1$ is utilized for testing then the data from all of the P2, P3, P4, and P5 positions will be held for training, and when P2 data is utilized for testing then the data from P1, P3, P4, and P5 will be held for training and so on. The classification error rates for this experiment are shown in Fig. 10 for the five testing positions at which four different classifiers were tested individually, i.e., one at a time. These results indicate a general enhancement on the classification results when training upon EMG data from multiple positions with an overall average of $8.85 \%, 9.62 \%, 9.34 \%$, and $8.86 \%$ for the LIBSVM, LDA, $k \mathrm{NN}$, and ELM respectively across the different limb positions. Such results indicate that EMG classification error is strongly dependent on limb position. This 


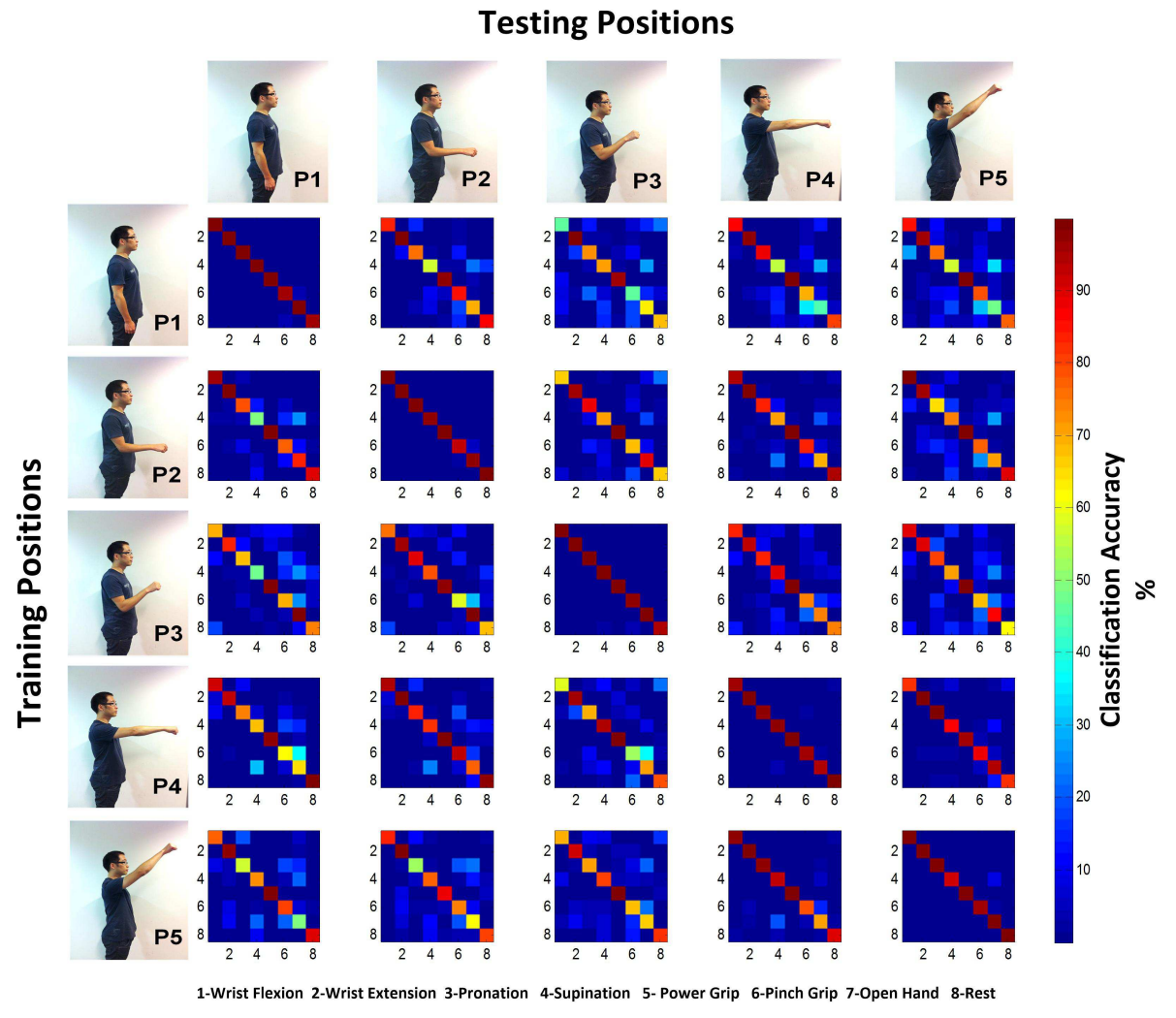

Figure 9: Confusion matrix plots across multiple limb positions using the LIBSVM classifier.

dependence may be attributable to variations in muscle recruitment (for limb stabilization due to gravitational forces), the force-length relationship of the muscle, and changes in the musculo-tendon lever arm, which all depend on joint angles. As the classifiers are now trained on different samples reflecting the aforementioned variations then the classification error rates were generally decreased upon the error rates resulting from training on individual limb position. The confusion matrices plots were also computed as shown in Fig.11 that further supports the previous results on the significance of having the training data collected from multiple limb positions.

However, it should be also mentioned here that P3 was the only position exhibiting relatively high error rates in comparison to the other positions. This in turn might be attributed to the angle of the limb positions which might be causing an electrode shift that deteriorates the classification error 


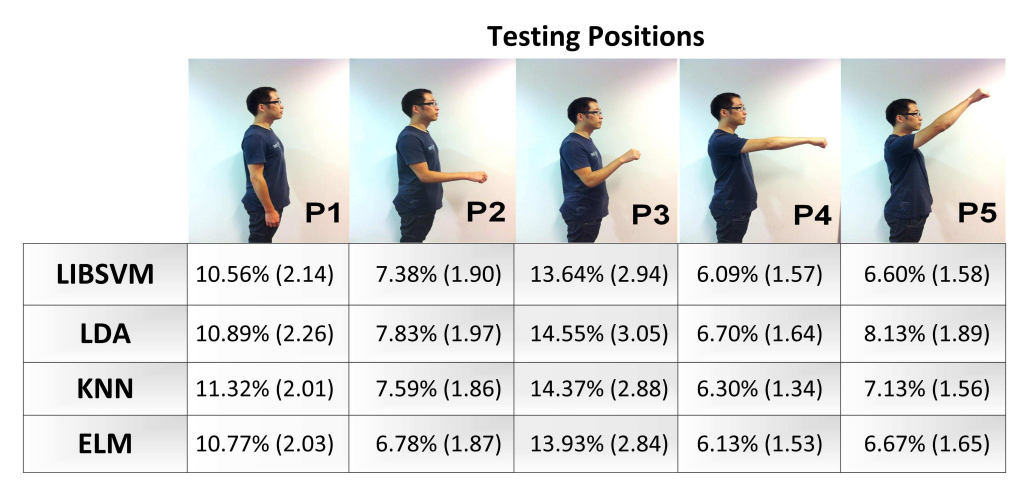

Figure 10: Classification error rates when training on EMG data from all positions, except the one being tested on. These results are the average across all movements and all subjects with standard error of mean between brackets for all different classifiers. The figure presents the LIBSVM results first schematically and then tabulate the results from all classifiers.
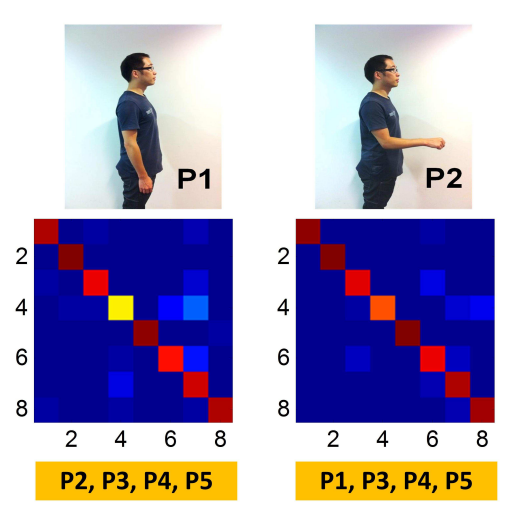

\section{Testing Positions}
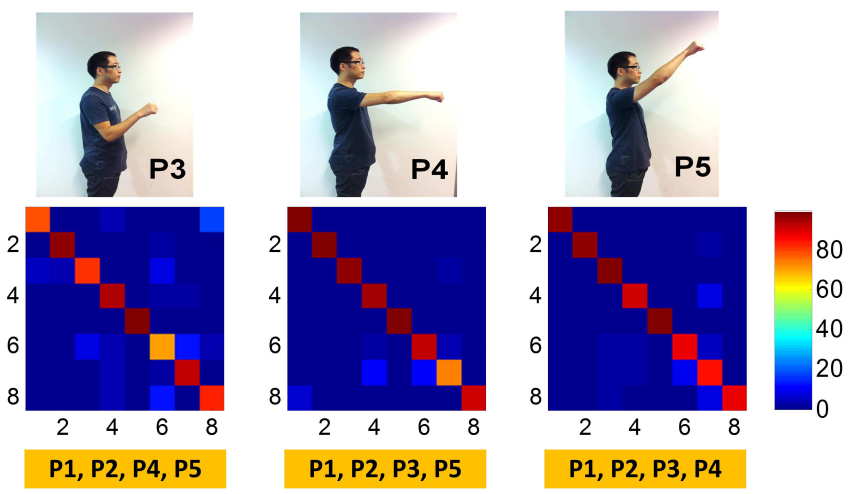

\section{Training Positions}

Figure 11: Confusion matrix plots across multiple limb positions when training on data from multiple limb positions and testing on individual limb positions, unseen during training, using the LIBSVM classifier.

rates. The results also indicate that the classification system can perform well on testing on EMG data from P2 and P4 while being trained on EMG data from other positions. Since training in multiple positions can be cumbersome for the end user, it is desirable to reduce the number of training positions to as much as possible. To confirm the possibility of training on fewer limb positions, another experiment was carried on in which we train the LIBSVM 
classifier with the data from P1, P3, and P5 only, while testing the classifier on data from P2 and P4. The average error rate in this case is $7.31 \% \pm 1.54$ with the average confusion matrix shown in Fig.12. Thus, one can train the system on EMG data from P1, P3, and P5 only as the classifier was able to generalize well on the rest of the positions. A real-time demonstration was also prepared and is available for the readers ${ }^{4}$. The demo clearly shows the good performance of the proposed EMG pattern recognition system when trained on the data from P1, P3, and P5 and being tested at any possible limb position in real-time.

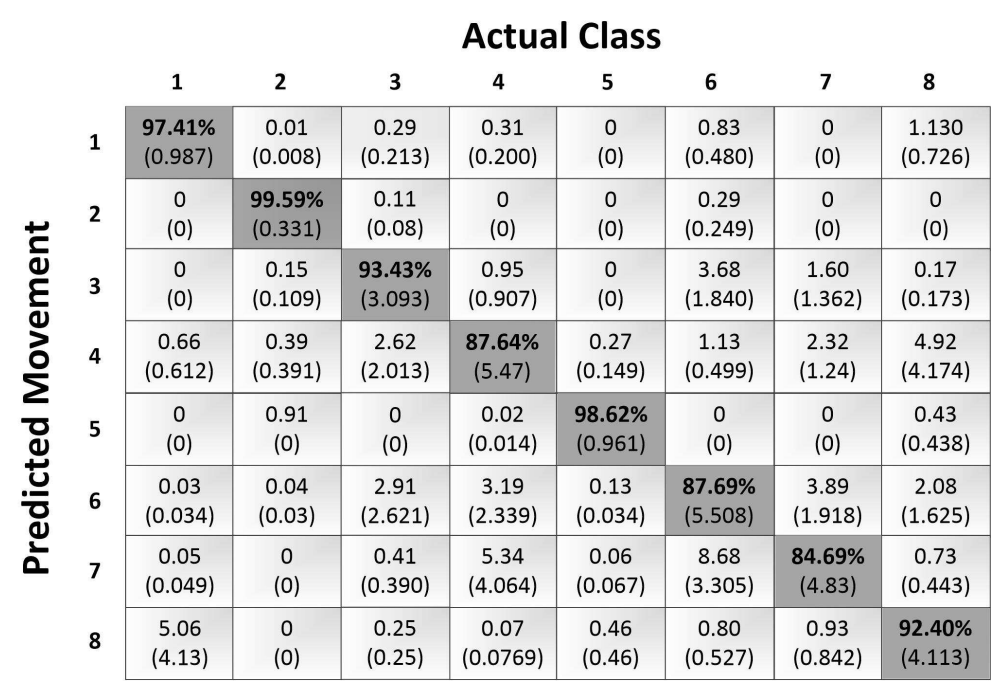

Figure 12: Confusion matrix averaged across multiple subjects while training the LIBSVM classifier on the data from P1, P3, and P5 and testing the classifier on features extracted from EMG data collected at P2 and P4. The that larger magnitudes or percentages in the main diagonal of the confusion matrix reflect the correct guesses, while the small values in the off-diagonal represent the incorrect guesses.

On the other hand, when including training data from all of the positions, the resulting classifiers were able to generalize in a better way on the unseen testing data from any position. In such a case, the six trials of collected data from each movement at each position were divided equally into three trials for training and three trials for testing, with the achieved error results reported in Fig. 13. In this case, the overall average classification error rates

\footnotetext{
${ }^{4}$ http: //www $\cdot$ youtube. $\operatorname{com} /$ watch?v=JRqXIv0xrNO\&feature=youtu be
} 
across all subjects and all movements dropped from $8.85 \%$ to $3.25 \%$ only when using the LIBSVM classifier indicating a significant enhancement in the results. An analysis of variance test with utilized to confirm the significance of the achieved results with a significance value of 0.05 which resulted in the corresponding $p$-value of 0.0058 , which in turn validates the significance of these results.

\section{Testing Positions}

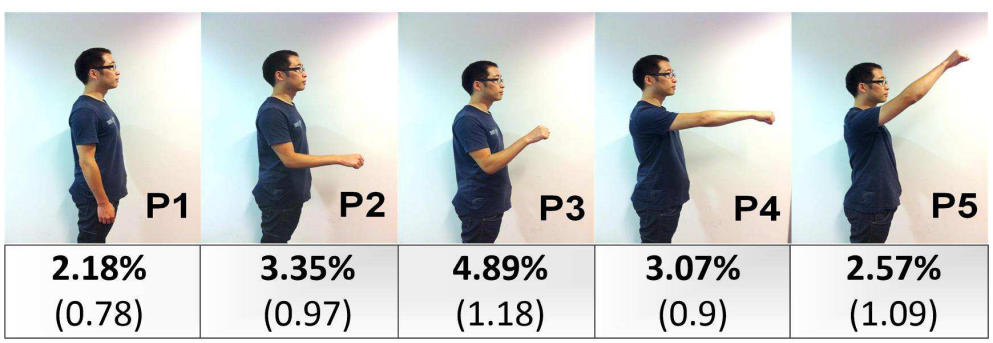

Training data from all positions

Figure 13: Classification error rates using the LIBSVM classifier when training on EMG data from all positions, including the one being tested on. These are the average across all movements and all subjects, with standard error of mean between brackets.

According to Geng et al. [66], the average EMG classification error rates when training on multiple limb positions for subjects with transradial amputations were significantly lower than that for intact upper limb subjects while using EMG. The authors suggested that the EMG signals acquired from an intact limb are more easily affected by limb position variation as subjects with intact limb have much more complex patterns of EMG recruitment than subjects with amputated limbs [66]. Given the aforementioned suggestion, the achieved error rates of $8.85 \%$ with the LIBSVM classifier should thus be considered acceptable, in a functional sense, for transradial amputees. However, such a claim would need further testing on amputees subjects. In the current work, the population of prosthesis users was limited, so for practical and ethical reasons the present method assessment using able-bodied subjects represents a necessary first step toward this ultimate goal. The next step of our research will be to validate the results by application to subjects with amputated limbs. 


\subsection{A Comparison with other methods}

In this part of the experiments, we test the performance of the proposed feature set, denoted as TDMOM, against other well-known feature extraction methods from the literature. These include: Hjorth parameters (denoted as Hjorth) [48, 49], reduced spectral moments by Vuskovic and Du (denoted as REDMOM) [32], time-domain features utilized by Fougner et al. (denoted as TD) [17], Barlow moments [67], the first four moments of the power spectral density PSD (denoted as PSDMOM), a combination of time-domain and Autoregressive model parameters (denoted as TDAR) [9], and the wavelet features represented by energy, variance and standard deviation of the coefficients of at each node of Symmlet-8 family tree with 7 levels decomposition. It should be mentioned here that that in order to provide a fair comparison with the other methods, then we have utilized the same windowing scheme, dimensionality reduction (OFNDA), classifier (LIBSVM), and post processing steps when the classifications errors with the traditional features mentioned above are used.

In this part of the experiments, we present two sets of performance comparison where in the first part, we train the classifier with data from only one limb position, as opposed to multiple positions, as this training scheme will remove the possible enhancement of the classifier by providing it with a general data set. In a similar way to computing the plots presented in Fig.8, we have calculated the inter-and-intra position error matrices for each of the aforementioned feature sets. The mean inter-position errors (this is the mean of the off-diagonal elements of the inter-position classification error matrices) were calculated as $22.58 \%, 23.6921 \%, 23.6266 \%, 26.1031 \%, 27.0694 \%$, $24.9905 \%, 25.5725 \%$, and $22.1959 \%$ for each of the TDMOM, Hjorth, REDMOM, TD, TDAR, Barlow, PSDMOM, and wavelet features respectively. These results indicate clearly that TDMOM and the wavelet features performed substantially better in comparison to the TD, TDAR, Barlow and PSDMOM and slightly better than REDMOM and Hjorth. This is an indication that both of the proposed TDMOM and the wavelet features tend to be more robust against the variation in limb position than other features. This is further confirmed by the next part of this experiment.

In the second part, we train the classifier on data from multiple limb positions and observe the generalization capability of the classifier when been tested on data from an unseen position that was not included in the training. In simpler words, training data was acquired from multiple limb positions, except from the position upon which the testing data was acquired from, 
i.e., training and testing data are from completely separate positions. As an example, when training on the data from P2, P3, P4, and P5 and testing on $\mathrm{P} 1$ the achieved error rates were $10.56 \%$ on average across all subjects, while training on the data from P1, P3, P4, and P5 and testing on the data from $\mathrm{P} 2$ resulted in an average error rate of $7.38 \%$ on average across all subjects, and so on for the rest of the positions with the results shown in Fig. 14. These results clearly show that the proposed feature set was able to compete with the other methods and achieve, on average across all subjects, lower classification error rates, except on P4 where wavelet features showed slightly lower error rates. The average error rates, across all positions, were also computed as $8.85 \%$ for our proposed moments, $12.06 \%$ for Hjorth parameters, $12.67 \%$ for REDMOM moments [32], $12.32 \%$ for TD features, and $12.73 \%$ for TDAR features, $11.69 \%$ for Barlow moments, $12.71 \%$ for PSDMOM, and $9.8 \%$ for wavelet features. These results further suggests that the performance of the Hjorth, RECMOM, TD, TDAR, Barlow, and PSDMOM features tend to be significantly lower than that of our proposed TDMOM and the wavelet features when the training data was acquired from multiple positions. This is justified by the confusion caused to the classifier by these features when these features were combined from multiple limb positions to form the training set. Finally, it should be also noted here that despite the close performance by our proposed method and that of the wavelet features, the computational complexity of our time domain features is much lower than that of the wavelet features. This in turn forms a factor that favours our proposed features upon that of the wavelet features.

In order to evaluate the statistical significance of the aforementioned classification error rates achieved by our proposed method in comparison to each of the other feature extraction methods, then we have utilized the well-known Student's t-test with Bonferroni correction. Bonferroni correction was utilized here to reduce the chance of committing a type-I error that is denoting the output from the test as significant while in reality it is not significant and vice versa. For a significance level of 0.05 , the achieved $p$-values for comparing our method against each of the other methods were $<<0.01$ indicating significant enhancements by our method, except that of the wavelet features, for which the later came out with a $p$-value of 0.08 indicating no significant differences between our method and the wavelet features. However, one important point that may result in favoring our method upon the wavelet feature extraction is the associated computational time required by the different methods. In specific, on a laptop with i7 processor operating 


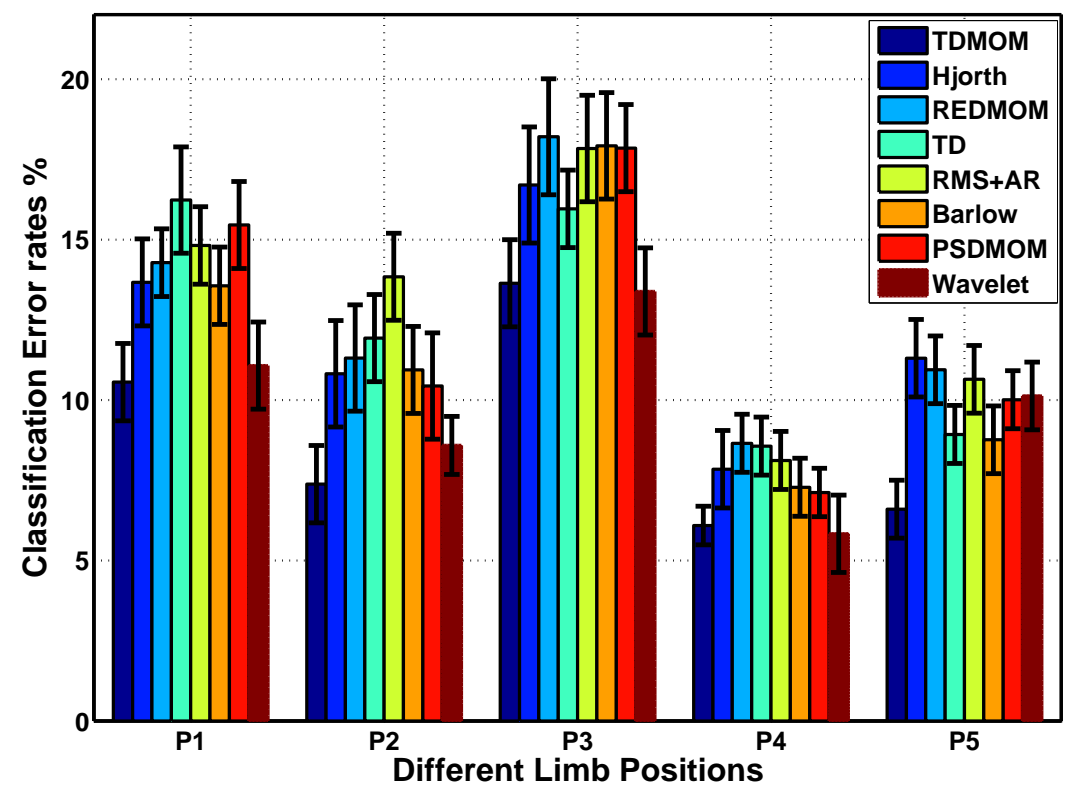

Figure 14: Different feature sets classification error rates when training on EMG data from all positions, except the one being tested on. These are the average across all movements and all subjects with bars indicating standard error.

at $1.73 \mathrm{GHz}$ with 4 cores and $16 \mathrm{~GB}$ of RAM running windows 8 and Matlab 2013 and for a record length of $100 \mathrm{msec}$ the proposed TDMOM required $0.56 \mathrm{msec}$ to compute the proposed features while the wavelet decomposition required $2.6 \mathrm{msec}$, i.e., an advantage of time reduction by a factor of (2.6$0.56) / 2.6^{*} 100=78.46 \%$. Finally it should be also mentioned here that our main argument is not to compete with the wavelet features, but to produce a time-domain implementation for the features that usually require a transformation to the frequency-domain while maintaining the accuracy. It is true that modern processors might be able to further reduce the computational time by both methods, however, with the advent in signal processing tool, further reducing the computational costs is always a desire for any real-time implementation.

\section{Conclusion}

The paper provided an investigation into the effect of upper-limb position on EMG pattern recognition by proposing a new feature set based on timedomain derivations of spectral moments. It was shown that based on Fourier 
transform time-frequency relationships, a set of spectral moments were derived directly from the time-domain which in turn reduced the computational complexity and formed a set of invariants to signal scaling, amplification and translation. The EMG data utilized in testing the proposed feature set was acquired from eleven subjects performing eight classes of hand movements at five different limb positions. Two sets of experiments were conducted involving off-line testing, with EMG records of movements held for 5 seconds each (with a sliding window approach of $100 \mathrm{msec}$ incremented by $25 \mathrm{msec}$ ), and an on-line test with a video demonstration also provided. The results indicated that by using the proposed features and training the classifier on EMG data from multiple limb positions it was possible for the classifier to generalize well upon EMG data from unseen positions with average error rates of up to $8.85 \%$ on average across all positions. The results also suggested that only three positions were required for training the classifier which in turn resulted in a reduced cost of data collection during training.

\section{References}

[1] B. Hudgins, P. Parker, and R. N. Scott RN, "A new strategy for multifunction myoelectric control", IEEE Transactions on Biomedical Engineering, vol. 40, no. 1, pp. 82-94, 1993.

[2] K. Englehart K and B. Hudgins, "A robust, real-time control scheme for multifunction myoelectric control", IEEE Transactions on Biomedical Engineering, vol. 50, no. 7, pp. 848-854, 2003.

[3] R. Merletti and P. A. Parker, "Electromyography Physiology, Engineering, and Noninvasive Applications", IEEE Press Series in Biomedical Engineering, John Wiley and Sons Inc, Hoboken, New Jersey, 2004.

[4] A. D. C. Chan, K. Englehart, B. Hudgins, and D. F. Lovely, "Hidden Markov model classification of myoelectric signals in speech", IEEE Engineering in Medicine and Biology Magazine, vol. 21, no. 4, pp.143-146, 2002 .

[5] E. J. Scheme, B. Hudgins, P. A. Parker, "Myoelectric Signal Classification for Phoneme-Based Speech Recognition", IEEE Transactions on Biomedical Engineering, vol. 54, no. 4, pp. 694-699, 2007. 
[6] T. S. Saponas, D. S. Tan, D. Morris, and R. Balakrishnan, "Demonstrating the feasibility of using forearm Electromyography for muscle-computer interfaces", In proceedings of the ACM Conference on Human Factors in Computing Systems (CHI), April 5-10, Florence, Italy, 2008.

[7] T. S. Saponas, D. S. Tan, D. Morris, J. Turner, and J. A. Landay, "Making muscle-computer interfaces more practical", In proceedings of the ACM Conference on Human Factors in Computing Systems (CHI), April 1015, Atlanta, Georgia, USA, 2010.

[8] D. Graupe, J. Salahi, and K. H. Kohn, "Multifunctional prosthesis and orthosis control via microcomputer identification of temporal pattern differences in single-site myoelectric signals", Journal of Biomedical Engineering, vol. 4, no. 1, pp. 17-22, 1982.

[9] M. A. Oskoei and H. Hu, "Myoelectric control systems-A survey", Biomedical Signal Processing and Control, vol. 2, no. 4, pp. 275-294, 2007.

[10] G. Li, "Electromyography pattern-recognition-based control of powered multifunctional upper-limb prostheses", In Joseph Mizrahi (Eds), Advances in Applied Electromyography, InTech, pp 99-116, 2011.

[11] B.A. Lock, "Design and Interactive Assessment of Continuous Multifunction Myoelectric Control Systems", Masters Thesis, University of New Brunswick, Fredericton, New Brunswick, 2005.

[12] L. Hargrove, K. Englehart, and B. Hudgins. "A training strategy to reduce classification degradation due to electrode displacements in pattern recognition based myoelectric control", Biomedical Signal Processing and Control, vol. 3, no. 2, pp. 175180, 2008.

[13] E. J. Scheme, K. B. Englehart, B. Hudgins, "Selective classification for improved robustness of myoelectric control under nonideal conditions", IEEE Transactions on Biomedical Engineering, vol. 58, no. 6, pp. 16981705, 2011.

[14] C. Cipriani, M. Controzzi, G, Kanitz, and R. Sassu, "The Effects of Weight and Inertia of the Prosthesis on the Sensitivity of Electromyographic Pattern Recognition in Relax State", Journal of Prosthetics and Orthotics, vol. 24, no. 2, pp. 86-92, 2012. 
[15] E. Scheme, A. Fougner, A. D. C. Chan, O. Stavdahl, and K. Englehart, "Examining the adverse effects of limb position on pattern recognition based myoelectric control", in Proceedings of Engineering in Medicine and Biology Society (EMBS) Conference, Buenos Aires, Sep., pp. 6337-6340, 2010 .

[16] L. Chen, Y. Geng, and G. Li, "Effect of Upper-Limb Positions on Motion Pattern Recognition Using Electromyography", In Proceedings of the 4th International Congress on Image and Signal Processing, pp. 139-142, 2011.

[17] A. Fougner, E. Scheme, A. D. C. Chan, K. Englehart, and . Stavdahl, "Resolving the Limb Position Effect in Myoelectric Pattern Recognition", IEEE Transactions on Neural Systems and Rehabilitation Engineering, vol. 19, no. 6, pp. 644-651, 2011.

[18] X. Zhang and P. Zhou, "High-Density Myoelectric Pattern Recognition Toward Improved Stroke Rehabilitation", IEEE Transactions on Biomedical Engineering, vol. 59, no. 6, pp. 1649-1657, 2012.

[19] N. Jiang, S. Dosen, K. R. Muller, and D. Farina, "Myoelectric control of artificial limbs- is there a need to change focus?", IEEE Signal Processing Magazine, vol. 29, no. 5, pp. 148-152, 2012.

[20] R. Boostani and M. H. Moradi, "Evaluation of the forearm EMG signal features for the control of a prosthetic hand", Physiological Measurement, vol. 24, pp. 309-319, 2003.

[21] B. Hannaford and S. Lehman, "Short time Fourier analysis of the Electromyogram: Fast movements and constant contraction", IEEE Transactions on Biomedical Engineering, vol. BME-33, no. 12, pp. 1173-1181, 1986.

[22] M. Zardoshti-Kermani, B. C. Wheeler, K. Badie, and R. M. Hashemi, "EMG feature evaluation for movement control of upper extremity prostheses", IEEE Transactions on Rehabilitation Engineering, vol. 3, no. 4, pp. 324-333, 1995.

[23] S. Du and M. Vuskovic, "Temporal vs. spectral approach to feature extraction from prehensile EMG signals", In Proceedings of the IEEE International Conference on Information Reuse and Integration, pp. 344-350, 2004 . 
[24] J. Rafiee, M.A. Rafiee, F. Yavari, and M.P. Schoen, "Feature extraction of forearm EMG signals for prosthetics", Expert Systems with Applications, vol. 38, no. 4, pp. 4058-4067, 2011.

[25] A. Phinyomark, P. Phukpattaranont, and C. Limsakul, "Feature reduction and selection for EMG signal classification", Expert Systems with Applications, vol. 39, no. 8, pp. 74207431, 2012.

[26] P. Shenoy, K. J. Miller, B. Crawford, R. P. N. Rao, "Online Electromyographic Control of a Robotic Prosthesis", IEEE Transactions on Biomedical Engineering, vol. 55, no. 3, pp. 1128-1135, 2008.

[27] A. Phinyomark, S. Hirunviriya, C. Limsakul, and P. Phukpattaranont, "Evaluation of EMG feature extraction for hand movement recognition based on Euclidean distance and standard deviation", In International Conference on Electrical Engineering/Electronics Computer Telecommunications and Information Technology (ECTI-CON), pp. 856-860, 2010.

[28] A. Fougner, . Stavdahl, P. J. Kyberd, Y. G. Losier, and P. A. Parker, "Control of Upper Limb Prostheses: Terminology and Proportional Myoelectric Control - A Review", IEEE Transactions on Neural Systems and Rehabilitation Engineering, DOI 10.1109/TNSRE.2012.2196711, 2012.

[29] J. U Chu, I. Moon, S. K. Kim, M. S. Mun, "Control of multifunction myoelectric hand using a real-time EMG pattern recognition", In IEEE/RSJ International Conference on Intelligent Robots and Systems (IROS), pp. 3511-3516, 2005.

[30] Y. Matsumura, M. Fukumi, Y. Mitsukura, "Hybrid EMG Recognition System by MDA and PCA", In Proceedings of the International Joint Conference on Neural Networks, IJCNN, pp. 5294-5300, 2006.

[31] A. R. Goge and A. D. C. Chan, "Investigating classification parameters for continuous myoelectrically controlled prostheses", In Proceedings of the 28th Conference of the Canadian Medical and Biological Engineering Soceity (CMBEC28), Quebec City, Canada, pp. 141-144, 2004.

[32] M. Vuskovic and S. Du, "Spectral moments for feature extraction from temporal signals", In International Conference on Intelligent Computing. August 23-26, HeFei, China, pp. 1063-1072, 2005. 
[33] A. Phinyomark, C. Limsakul, and P. Phukpattaranont, "A Novel Feature Extraction for Robust EMG Pattern Recognition", Journal of Computing, vol. 1, no. 1, pp. 2151-9617, 2009.

[34] S. Karlsson, Y. Jun, and M. Akay, "Time-frequency analysis of myoelectric signals during dynamic contractions: A comparative study", IEEE Transactions on Biomedical Engineering, vol. 47, no. 2, pp. 228-238, 2000.

[35] K. Englehart, "Signal representation for classification of the transient myoelectric signal", PhD dissertation, Department of Electrical and Computer Engineering, University of New Brunswick, New Brunswick, Canada, 1998.

[36] K. Englehart, B. Hudgins, P. A. and Parker, "A wavelet-based continuous classification scheme for multifunction myoelectric control", IEEE Transactions on Biomedical Engineering, vol. 48, no. 3, pp. 302-311, 2001.

[37] J. U. Chu, I. Moon, and M. S. Mun, "A supervised feature projection for real-time multifunction myoelectric hand control", In Proceedings of the 28th IEEE Engineering in Medicine and Biology Society (EMBS'06) Annual International Conference, New York City, USA, pp. 2417-2420, 2006 .

[38] G. Wang, Z. Wang, W. Chen, and J. Zhuang, "Classification of surface EMG signals using optimal wavelet packet method based on DaviesBouldin criterion", Medical and Biological Engineering and Computing, vol. 44, no. 10, pp. 865-872, 2007.

[39] R. N. Khushaba, A. Al-Jumaily, and A. Al-Ani, "Novel feature extraction method based on fuzzy entropy and wavelet packet transform for myoelectric control", In Proceedings of the International Symposium on Communications and Information Technologies (ISCIT), pp. 352-357, 2007.

[40] Z. Yan, Z. Wang, and H. Xie, "Joint application of rough set-based feature reduction and Fuzzy LS-SVM classifier in motion classification", Medical and Biological Engineering and Computing, vol. 46, no. 6, pp. 519-527, 2008.

[41] K. A. Farry, I. D. Walker, and R. G. Baraniuk, "Myoelectric teleoperation of a complex robotic hand", IEEE Transactions on Robotics and Automation, vol. 12, no. 5, pp. 775-787, 1996. 
[42] S. M. Kay, "Modern Spectral Estimation: theory and Application", Englewood Cliffs, NJ, Prentice Hall, 1998.

[43] S. Du, "Feature extraction for classification prehensile Electromyography patterns", Master thesis, Department of Computer Science, San Diego State University, 2003.

[44] Y. H. Huang, K. Englehart, B. S. Hudgins, and A. D. C. Chan, "A Gaussian mixture model based classification scheme for myoelectric control of powered upper limb prostheses", IEEE Transactions on Biomedical Engineering, vol. 52, no. 11, pp. 1801-1811, 2005.

[45] R. N. Khushaba, A. Al-Ani, A. Al-Jumaily, "Orthogonal Fuzzy Neighborhood Discriminant Analysis for Multifunction Myoelectric Hand Control", IEEE Transactions on Biomedical Engineering, vol. 57, no. 6, pp. 1410-1419, 2010.

[46] L. J. Hargrove, E. J. Scheme, K. B. Englehart, B. S. Hudgins, "Multiple Binary Classifications via Linear Discriminant Analysis for Improved Controllability of a Powered Prosthesis", IEEE Transactions on Neural Systems and Rehabilitation Engineering, vol. 18, no. 1, pp. 49-57, 2010.

[47] H. Huang, T. A. Kuiken, and R. D. Lipschutz, "A Strategy for Identifying Locomotion Modes Using Surface Electromyography", IEEE Transactions on Biomedical Engineering, vol. 56, no. 1, pp. 65-73, 2009.

[48] B. Hjorth, "EEG analysis based on time domain properties", Electroencephalography and Clinical Neurophysiology, vol. 29, no. 3, pp. 306-310, 1970.

[49] R. N. Khushaba, S. Kodagoa, D. Liu, and G. Dissanayake, "Electromyogram (EMG) based Fingers Movement Recognition Using Neighborhood Preserving Analysis with QR-Decomposition", In proceedings of the International Conference on Intelligent Sensors, Sensor Networks and Information Processing, Adelaide, Australia, pp. 100-105, 2011.

[50] J. Flusser, T. Suk, and B. Zitova, "Moments and moment invariants in pattern recognition", John-Wiley and Sons Ltd Publication, United Kingdom, 2009. 
[51] C. T. Lin, R. C. Wu, T. P. Jung, S. F. Liang, and T. Y. Huang, "Estimating alertness level based on EEG spectrum analysis," EURASIP Journal of Applied Signal Processing, vol. 2005, no. 19, pp. 31653174, 2005.

[52] C. T. Lin, R. C. Wu, S. F. Liang, W. H. Chao, Y. J. Chen, and T. P. Jung, "EEG-based drowsiness estimation for safety driving using independent component analysis," IEEE Transactions on Circuits and SystemsI, Reg. Papers, vol. 52, no. 12, pp. 27262738, 2005.

[53] D. Tkach, H. Huang, and T. A Kuiken, "Study of stability of timedomain features for electromyographic pattern recognition", Journal of NeuroEngineering and Rehabilitation, vol. 7, no. 21, 2010.

[54] J. V. Basmajian and C. J. De Luca, "Muscles Alive (5th edition)", Williams and Wilkins, Baltimore, MD, 1985.

[55] B. Vigreux, J. C. Cnockaert, and E. Pertuzon, "Factors influencing quantified surface EMGs", European Journal of Applied Physiology and Occupational Physiology, vol. 41, no. 2, pp 119-129, 1979.

[56] K. Sahlin, M. Tonkonogi,and K. Soderlund, "Energy supply and muscle fatigue in humans", Acta Physiologica Scandinavica, vol. 162, no. 3, pp. 261-266, 1998.

[57] T. Dirlik, "Application of computers in fatigue", PhD Thesis, Department of Engineering, University of Warwick Coventry, England, 1985.

[58] J. P. Bello, L. Daudet, S. Abdallah, C. Duxbury, M. Davies, and M. B. Sandler, "A Tutorial on Onset Detection in Music Signals", IEEE Transactions on Speech and Audio Processing, vol. 13, no. 5, pp. 1035-1047, 2005 .

[59] L.B. White and B. Boashash, "Cross Spectral Analysis of NonStationary Processes", IEEE Transactions on Information Theory, vol. 36, no. 4, pp. 830-835, 1990.

[60] J. O. Smith, "Mathematics of the Discrete Fourier Transform (DFT) with Audio Applications, Second Edition", W3K Publishing, ISBN 978-09745607-4-8, 2007 
[61] S. Theodoridis and K. Koutroumbas, "Pattern Recognition", Fourth Edition. Academic Press, Elsevier, UK, 2009.

[62] T. R. Farrell and R. F. Weir, "The optimal controller delay for myoelectric prostheses", IEEE Transactions on Neural Systems and Rehabilitation Engineering, vol. 15, no. pp. 111-118, 2007.

[63] M. A. Oskoei, and H. Hu, "Support Vector Machine-Based Classification Scheme for Myoelectric Control Applied to Upper Limb", IEEE Transactions on Biomedical Engineering, vol. 55, no. 8, 2008.

[64] G.-B. Huang, H. Zhou, X. Ding, and R. Zhang, "Extreme learning machine for regression and multiclass classification", IEEE Transactions on Systems, Man, and Cybernetics, Part B: Cybernetics, vol. 42, no. 2, pp. 513-529, 2012.

[65] R. N. Khushaba, S. Kodagoda, M. Takruri, and G. Dissanayake, "Toward improved control of prosthetic fingers using surface electromyogram (EMG) signals", Expert Systems with Applications, vol. 39, pp. 1073110738, 2012.

[66] Y. Geng, L. Chen, L. Tian, G. Li, "Comparison of electromyography and mechanomyogram in control of prosthetic system in multiple limb positions", In Proceedings of IEEE-EMBS International Conference on Biomedical and Health Informatics (BHI), pp. 788-791, 2012.

[67] I. I. Goncharova and J. S. Barlow, "Changes in EEG mean frequency and spectral purity during spontaneous alpha blocking", Electroencephalography and Clinical Neurophysiology, vol. 76, no. 3, pp. 197-204, 1990. 